\title{
Differential dynamics of early stages of platelet adhesion and spreading on collagen IV- and fibrinogen-coated surfaces
}

\section{[version 1; peer review: 3 approved]}

\author{
Melanie B. Horev (D1), Yishaia Zabary (iD), Revital Zarka1, Simona Sorrentino3, \\ Ohad Medalia3, Assaf Zaritsky (D2), Benjamin Geiger (iD) \\ ${ }^{1}$ Department of Immunology, Weizmann Institute of Science, Rehovot, Rehovot, 76100, Israel \\ 2Department of Software and Information Systems Engineering, Ben-Gurion University of the Negev, Beer-Sheva, 84105, Israel \\ ${ }^{3}$ Department of Biochemistry, University of Zurich, Zurich, $\mathrm{CH}-8057$, Switzerland
}

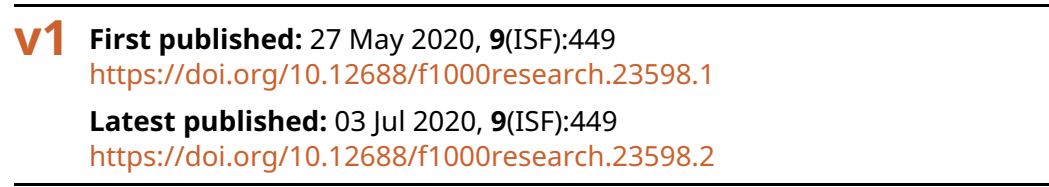

\section{Abstract}

Background: Upon wound formation, platelets adhere to the neighboring extracellular matrix and spread on it, a process which is critical for physiological wound healing. Multiple external factors, such as the molecular composition of the environment and its mechanical properties, play a key role in this process and direct its speed and outcome.

Methods: We combined live cell imaging, quantitative interference reflection microscopy and cryo-electron tomography to characterize, at a single platelet level, the differential spatiotemporal dynamics of the adhesion process to fibrinogen- and collagen IV-functionalized surfaces.

Results: Initially, platelets sense both substrates by transient rapid extensions of filopodia. On collagen IV, a short-term phase of filopodial extension is followed by lamellipodia-based spreading. This transition is preceded by the extension of a single or couple of microtubules into the platelet's periphery and their apparent insertion into the core of the filopodia. On fibrinogen surfaces, the filopodia-tolamellipodia transition was partial and microtubule extension was not observed leading to limited spreading, which could be restored by manganese or thrombin.

Conclusions: Based on these results, we propose that interaction with collagen IV stimulate platelets to extend microtubules to peripheral filopodia, which in turn, enhances filopodial-to-lamellipodial transition and overall lamellipodia-based spreading. Fibrinogen, on the other hand, fails to induce these early microtubule extensions, leading to full lamellipodia spreading in only a fraction of the seeded platelets. We further suggest that activation of integrin $\operatorname{aIIb} \beta 3$ is essential for filopodial-to-lamellipodial transition, based on the capacity of integrin activators to enhance lamellipodia spreading on fibrinogen.
Open Peer Review

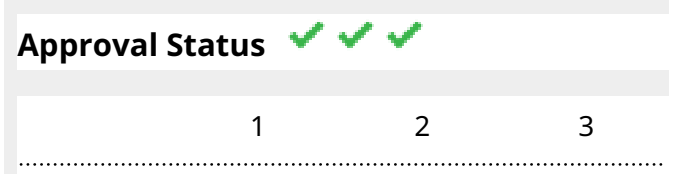

version 2

(revision)

view

$03 \mathrm{Jul} 2020$

version 1

27 May 2020
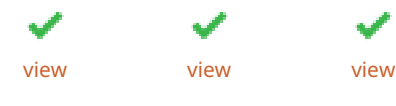

1. Sandra Citi iD, University of Geneva,

Geneva, Switzerland

2. Kenneth Yamada (iD), National Institute of Dental and Craniofacial Research, Bethesda, USA

3. Ulrich Schwarz $(D)$, Heidelberg University, Heidelberg, Germany

Any reports and responses or comments on the article can be found at the end of the article. 


\section{Keywords}

Platelet spreading, Microtubules, Integrin aIIbß3, Type IV collagen,

Fibrinogen, Interference Reflection Microscopy (IRM), Live cell imaging

\section{Corresponding author: Benjamin Geiger (benny.geiger@weizmann.ac.il)}

Author roles: Horev MB: Data Curation, Formal Analysis, Investigation, Methodology, Software, Validation, Visualization, Writing Original Draft Preparation, Writing - Review \& Editing; Zabary Y: Investigation, Methodology, Software, Visualization, Writing - Review \& Editing; Zarka R: Data Curation, Formal Analysis, Investigation, Software, Visualization; Sorrentino S: Data Curation, Investigation, Software, Visualization; Medalia O: Data Curation, Investigation, Software, Visualization; Zaritsky A: Investigation, Methodology, Software, Supervision, Visualization, Writing - Original Draft Preparation; Geiger B: Conceptualization, Data Curation, Funding Acquisition, Investigation, Project Administration, Resources, Supervision, Writing - Original Draft Preparation, Writing - Review \& Editing

Competing interests: No competing interests were disclosed.

Grant information: AZ is supported by the Israeli Council for Higher Education (CHE) via Data Science Research Center, Ben-Gurion University of the Negev. The research was supported in part from a grant from The Israel Science Foundation (ISF).

The funders had no role in study design, data collection and analysis, decision to publish, or preparation of the manuscript.

Copyright: $\odot 2020$ Horev MB et al. This is an open access article distributed under the terms of the Creative Commons Attribution License, which permits unrestricted use, distribution, and reproduction in any medium, provided the original work is properly cited.

How to cite this article: Horev MB, Zabary $Y$, Zarka $R$ et al. Differential dynamics of early stages of platelet adhesion and spreading on collagen IV- and fibrinogen-coated surfaces [version 1; peer review: 3 approved] F1000Research 2020, 9(ISF):449

https://doi.org/10.12688/f1000research.23598.1

First published: 27 May 2020, 9(ISF):449 https://doi.org/10.12688/f1000research.23598.1 


\section{Introduction}

Mammalian platelets are small, anucleated megakaryocytes fragments that circulate in the bloodstream as single, smoothsurfaced discoid structures. Upon vascular injury, platelets are recruited to the wound, where they initiate the formation on the primary hemostatic plug, catalyze fibrin formation and facilitate wound healing. Their adhesion and subsequent spreading at the exposed extracellular matrix (ECM) play a key role in the healing process, which is modulated by a variety of external environmental factors such as additional plasma components, other ECM constituents, platelet-derived secreted factors and their specific membrane receptors ${ }^{1-4}$. In addition to the molecular composition of the environment, its physical properties, such as rigidity, topography and shear forces generated by the blood flow, also influence the platelets adhesion and spreading process.

These molecular interactions of the platelets with the microenvironment are governed by specific adhesion receptors, such as $\alpha \operatorname{IIb} \beta 3$ integrin, which bind to fibrinogen and participated in the formation of the fibrin clot, and $\alpha 2 \beta 1$ and GP-VI, that mediate platelet adhesion to collagen IV $^{5-9}$. The molecular and physical cross-talk between platelets and their microenvironment was extensively investigated ${ }^{10-14}$, yet, given the complexity of the signaling pathways involved and their rapid activation, a comprehensive understanding of the platelet adhesion and activation process is still limited.

Previous studies have shown that platelets that are stimulated by a variety of agonists undergo radical morphological changes. The discoid shape, which is largely maintained by the circumferential marginal band of microtubules ${ }^{15-17}$, is affected by the agonists activation, and the cells acquire a spherical shape, extend filopodia and sheet-like lamellipodia ${ }^{18-20}$. The general process of platelet spreading on different surfaces was documented, yet how the platelets differentially respond to specific adhesive ligands is still unclear.

In this study, we compared the adhesion and spreading dynamics of isolated platelets on two distinct and physiologicallyrelevant ligands, namely collagen IV and fibrinogen, along a broad temporal scale, ranging from seconds to hours. We found that platelets in platelets-rich plasma (PRP-PL), and washed platelets (PLT) extend filopodia within a few seconds after reaching the close vicinity of fibrinogen or collagen IV surfaces, while still translocating along the surfaces. Stable adhesion to collagen IV or fibrinogen occurred roughly within a minute after filopodia extension, yet the mode of "immobilization" is clearly distinct; on fibrinogen the duration of "filopodial spreading" was shorter than on collagen IV (less than a minute, compared to $\sim 2-3$ minutes) and the onset of lamellipodia formation was apparent earlier ( 1 minute, compared to over 2-3 minutes on collagen IV). It was nevertheless noted that only a fraction of the fibrinogen-adherent PRP-PLs proceeded to the lamellipodial spreading stage.

Unexpectedly, the delayed lamellipodial spreading on collagen IV (but not on fibrinogen) was commonly preceded by an extension of a single or a couple of microtubules from the circumferential marginal band to the platelet periphery, that penetrated into the core of peripheral filopodia. These results suggest that microtubule extension to the periphery of collagen IV-adherent platelets might trigger filopodia-to-lamellipodia transition, and promote platelet spreading.

\section{Methods}

Preparation of fibrinogen and type IV collagen surfaces

MaTek dishes were incubated with either $25 \mu \mathrm{g} / \mathrm{ml}$ human collagen IV (Sigma-Aldrich, Israel) in sterile PBS or with $50 \mu \mathrm{g} / \mathrm{ml}$ human fibrinogen (Sigma-Aldrich, Israel) in sterile PBS, overnight at $4 C^{0}$. The human fibrinogen was rinsed twice in sterile PBS. Finally, blocking with BSA solution in sterile PBS (1mg/ml, $15 \mathrm{~min}$ incubation) was conducted, to reduce non- specific platelet attachment, followed by washing with PBS and with Tyrode's buffer. The collagen IV was washed with DMEM- no additives (Sigma-Aldrich, Israel) for $3 \times 30 \mathrm{~min}$. To reduce acidity, it was then washed two times with Tyrode's buffer. Both collagen IV and fibrinogen MaTek plates were subsided in Tyrode's-HEPES buffer $\mathrm{pH} 7.4$ containing $5 \mathrm{mM}$ dextrose/glucose.

\section{Preparation of platelets}

Human platelets in platelet rich plasma (PRP-PL) were produced from freshly drawn blood of different healthy donors specifically for this study. As no Helsinki approval is required for such experiments, we have worked under the Weizmann Institute of Science Institutional Review Board (IRB) informed consent form, signed by each of the volunteers. The IRB application (375-1) was approved by the committee members.

The blood was collected into PT vacutainer tubes (containing sodium citrate). After $10 \mathrm{~min}$ of incubation at RT, the blood was centrifuged at $800 \mathrm{rpm}$ for $10 \mathrm{~min}$, and the supernatant, containing platelets and plasma was collected. Human washed platelets (PLT) were produced from freshly drawn blood of different healthy donors. The blood was collected into PT vacutainer tubes (containing sodium citrate). After $10 \mathrm{~min}$ of incubation at RT, the blood was centrifuged at $800 \mathrm{rpm}$ for $10 \mathrm{~min}$, and the supernatant, containing platelets and plasma was collected. Then an equal volume of Tyrode's solution $\mathrm{pH}$ 6.5 containing $5 \mathrm{mM}$ dextrose, $0.2 \mu \mathrm{g} / \mathrm{mL} \mathrm{PGE}_{1}$ and $1.0 \mathrm{U} / \mathrm{mL}$ apyrase was added and incubated for $5 \mathrm{~min}$ at RT, then the diluted PRP was centrifuged at $1500 \mathrm{rpm}$ for $10 \mathrm{~min}$ to precipitate platelets. The supernatant was discarded and the pellet was re-suspended in Tyrode's solution, same volume as the discarded, at $\mathrm{pH} 6.5$ containing $5 \mathrm{mM}$ dextrose, $0.2 \mu \mathrm{g} / \mathrm{mL}$ $\mathrm{PGE}_{1}$ and $0.5 \mathrm{U} / \mathrm{mL}$ apyrase. The solution was incubated for $5 \mathrm{~min}$ at RT. Then it was centrifuged at $1500 \mathrm{rpm}$ for $10 \mathrm{~min}$. The supernatant was discarded and the pellet re-suspended in Tyrode's-HEPES buffer $\mathrm{pH} 7.4$ containing $5 \mathrm{mM}$ dextrose the same volume as the original PRP volume. The platelets were kept for 30-60 min at RT prior to use.

\section{Seeding of platelets on fibrinogen and collagen IV surfaces} for IRM

Fresh PRP-PL or PLT were seeded $\left(10-15 \times 10^{6}\right)$ on collagen IV and fibrinogen MaTek plates directly in the microscope 
environmental chamber at $37^{\circ} \mathrm{C}$ in humidified atmosphere of $5 \%$ $\mathrm{CO}_{2}$ and $95 \%$ air.

\section{Live-cell imaging and Interference Reflection Microscopy (IRM)}

PRP-PL and PLT attachment and spreading process on the surfaces was monitored in real time using the DeltaVision Elite $^{\circledR}$ system, running softWoRx 6.0. As the system is equipped with an environmental box, PRPs in Tyrode's solution were seeded directly onto the fibrinogen and collagen IV surfaces in the microscope environmental chamber (temperature- and $\mathrm{CO}_{2}$-controlled). Interference reflection contrast (IRM) timelapse imaging of several fields per specimen was carried out using 100x/1.3 oil objective, at 5 seconds between frames. IRM, phase-contrast microscopy, and differential interference contrast (DIC) microscopy still imaging were used with a 100X objective (NA 1.3 and 1.4), a 60X (NA 1.42) and a 20X (NA 0.85) objective.

\section{Spatiotemporal quantitative characterization of platelet spreading}

Single platelets were manually selected in the field-of-view. Since we were interested in minimizing the effects of neighboring platelets, we selected platelets that spread the earliest in their vicinity. For each platelet, we manually annotated a bounding box that captured the full spread platelet and recorded time points for the characterized stages in platelet spreading (defined in Results): onset, or initial appearance (time $=0$ ); onset of filopodia; and lamellipodia spreading.

To visualize and quantify the change in the local focal plane during platelet spreading, we developed custom software using Matlab and Python (Software availability ${ }^{21}$ ). We defined the focal activity map as the time-derivative of a platelet's IRM intensity. This was calculated for each time point as $\Delta \mathrm{IRM}_{\mathrm{t}}=\mathrm{IRM}_{\mathrm{t}}-\mathrm{IRM}_{\mathrm{t}+1}$. Local instantaneous attachment was encoded with positive values (time point $t+1$ is darker - closer to the substrate), while local instantaneous detachment corresponded to negative values (time point $t+1$ is brighter - further from the substrate). The focal activity map provides intuitive visual information on the platelet's spatiotemporal focal-dynamics.

The integrated tapping activity of a platelet in a given frame in the image sequence was defined as the accumulation of all local alterations in focal plane in both attachment of detachment events. This measure was calculated as the average value of the absolute $\Delta$ IRM image pixels: $\Sigma_{i}\left|I R M_{t}\right|_{i}$, where $i$ spans the platelet's bounding-box's pixels, and $\left|I R M_{t}\right|_{i}$ is the corresponding pixel's intensity (see Figure 2B). Importantly, this measure is relative; for example, more background pixels in a bounding box will dampen the magnitude regardless of the actual platelet activity.

To follow the spreading process, we quantified the pixels that were touching or very close to the surface. We pooled all IRM pixel intensities across a platelet time-lapse sequence and used the Otsu algorithm ${ }^{22}$ to define a threshold that labels each pixel at every time point into 'foreground' or 'background'. 'Foreground' pixels had low IRM intensities and were thus touching or very close to the surface, we termed these pixels as "interacting with the surface". 'Background' pixels had higher IRM intensities and marked local regions that did not touch the substrate. We measured the number of pixels interacting with the surface over time during the spreading process (see Figure 2C). For each pixel, at each time frame, we accumulated the number of transitions from interaction to not-interacting with the surface from time 0 and until that time point (see Figure 2D).

To examine the spatiotemporal statistics of a platelet spreading, we identified a subgroup of active pixels. First, we manually marked a background region, an empty region close to the platelet, collected and pooled $\triangle I R M$ pixel intensities in the background throughout the platelet spreading process. Second, we used the accumulated background pixel intensity statistics to define thresholds of one standard deviation above or below the mean background pixel $\triangle I R M$ intensity. The subgroup of pixels that exceeded these $\triangle I R M$ thresholds were the more active pixels in terms of attaching/detaching activity. We calculated the fraction of active pixels that attached or detached for each time frame (see Figure $3 \mathrm{~A}-\mathrm{C}$ ) and then overlaid the active pixels color-coded as attaching or detaching back to the image plane (see Figure 3D and E).

\section{Treatments}

$\mathrm{Mn}^{2+}(250 \mu \mathrm{M})$, molecule sn528 ( $\alpha \operatorname{IIb} \beta 3$ integrin inhibitor; $10 \mu \mathrm{M})$ or thrombin $(0.12 \mathrm{mg} / \mathrm{ml})$ were added directly to the dishes containing fibrinogen or collagen IV in Tyrode's-HEPES buffer $\mathrm{pH} 7.4$ containing $5 \mathrm{mM}$ dextrose simultaneously as the fresh PRP-PL or PLT, which were seeded $\left(10-15 \times 10^{6}\right)$ in the microscope at $37^{\circ} \mathrm{C}$ in humidified atmosphere of $5 \% \mathrm{CO}_{2}$ and $95 \%$ air

\section{Chemical fixation of platelets in solution}

The platelets were seeded for 10-20 $\mathrm{min}$ on fibrinogen or collagen IV. Subsequently, they were fixed by Karnovsky fixative (2\% paraformaldehyde, $2.5 \%$ glutaraldehyde). The solution was shaken well and incubated for $30 \mathrm{~min}$. Then glycerol was added, spun down gently, and washed with PBS. The platelets were then suspended in PBS and glycerol (1:9), and the solution closed between coverslip and slide and imaged by phase contrast microscopy.

\section{Treatment with Nocodazole}

Nocodazole $(10,15$ or $20 \mu \mathrm{M})$ was added to the platelets in solution immediately before seeding the platelets in the dish containing collagen IV in Tyrode's-HEPES buffer, pH 7.4, containing $5 \mathrm{mM}$ dextrose. Platelets were seeded $\left(10-15 \times 10^{6}\right)$ in the microscope at $37^{\circ} \mathrm{C}$ in a humidified atmosphere of $5 \% \mathrm{CO}_{2}$ and $95 \%$ air.

\section{Treatment with Von Willebrand factor (VWF)}

Von Willebrand factor (2400 IU) Heamate P 1000 (CSL Behring, GmbH, Germany) was used in different concentrations $(1: 2,1: 5,1: 10)$, all yielding the same results. VWF was added directly to the dish containing collagen IV in Tyrode's-HEPES 
buffer, $\mathrm{pH} 7.4$, containing $5 \mathrm{mM}$ dextrose, simultaneously with the fresh PLT, which were seeded $\left(10-15 \times 10^{6}\right)$ in the microscope at $37^{\circ} \mathrm{C}$ in a humidified atmosphere of $5 \% \mathrm{CO}_{2}$ and $95 \%$ air.

\section{Cryo-electron tomography}

Platelets were seeded on collagen IV functionalized gold/silicon grids (R 1/4, 200 mesh, Quantifoli, Jena Germany). The grids were then vitrified by plunge freezing in liquid ethane and then immediately stored in liquid nitrogen until imaging. Data acquisition was performed using a FEI Titan Krios equipped with a quantum energy filter and a K2 Summit direct electron detector (Gatan, Pleasanton, USA). Tomograms were acquired with a magnification of $42,000 \times$ corresponding to a pixel size of $0.34 \mathrm{~nm}$. The cumulative electron dose was $\sim 70$ electrons per ångström ${ }^{2}$. Tomograms were reconstructed with the TOM toolbox software package ${ }^{23}$.

\section{Data analysis}

Statistical calculations were performed using Excel (version 14.6.8, 2011) and a STATISTICA ${ }^{\circledR}$ (version 13.1) software package, using the non-parametric Wilcoxon rank-sum test.

Image analysis was performed using MATLAB (version 9.3), Amira/Avizo (version 3.1), motion correction software, and Fiji (ImageJ).

\section{Results}

We chose to study two preparations of platelets, isolated from human donor blood: washed platelets (termed here PLT) and platelets in platelet-rich-plasma (termed PRP-PL). To create and test physiologically relevant environments, we plated platelets on tissue culture surfaces coated with either fibrinogen, an integral component of the blood clot; or collagen IV, a basement membrane molecule that platelets adhere to upon vessel injury. It is noted that this adhesive set-up is not entirely 'clean', as platelets secrete multiple biologically active molecules (e.g. fibrinogen). Furthermore plasma components in the PRP-PL may affect the experiments (for further discussion, see below). In this study, we have employed real time microscopy (primarily interference reflection optics (IRM) or differential interference contrast (DIC) for monitoring platelet-substrate interaction.

Temporal steps in platelets' interactions with collagen IV and fibrinogen surfaces in a time scale of seconds to hours Pre-immobilization: Early events of the interaction of platelets with collagen IV-coated surfaces were visualized using live DIC imaging. With the microscope focused on the adhesive surface, we observed platelets that were located at the vicinity of the substrate, whose translocation was substantially attenuated relative to those still floating freely in the medium. Such loose interactions with the surface were noted within 15-25 seconds after reaching the substrate focal level (Figure 1A). We detected an active extension of "ventral filopodia" that was apparent as soon as 10 seconds after the platelet reached the surface (Figure 1A, black arrow inset) and persisted until the platelet became immotile. Upon turning immotile, the platelet continued to spread by extending stable substrate-attached filopodia, followed by lamellipodia, until reaching full spreading after 20-30 minutes (Figure 1A). We confirmed that some platelets extended filopodial extensions while being motile on collagen IV- or on fibrinogen-coated surfaces with chemical fixation and phase contrast microscopy imaging (Figure $1 \mathrm{~B}-\mathrm{C}$, arrows). These results confirmed the existence of a filopodial extension prior to platelets immobilization and attachment to the substrate.

Post immobilization, filopodial spreading: Platelets on collagen IV became immotile and attached to the surface at a faster pace than platelets on fibrinogen (Figure 1D). These results suggest that upon the onset of platelet immobilization and spreading, platelets can extend filopodial protrusions on both collagen IV and fibrinogen surfaces, but that platelet spreading is more effective on collagen IV surfaces.

Post immobilization, lamellipodial spreading: The filopodial spreading, which was initiated still in the "mobile phase", was followed by massive lamellipodial spreading ("filopodial-lamellipodial transition"), which was noted in the PRP-PL/fibrinogen system in $\sim 55 \%$ of the platelets at about 1 minute after the first interaction with the surface. The onset of the filopodial-lamellipodial transition in the PLT/collagen IV system was delayed ( $\sim 2-3$ minutes) yet was apparent in a larger proportion of the platelet population $\sim 75 \%$ (see results below).

Live cell monitoring of the differential dynamic interactions of platelets with collagen IV and fibrinogen surfaces

To further characterize the spatiotemporal dynamics involved in platelet adhesion to collagen IV-coated surfaces, we used live cell IRM, where the light intensity is negatively correlated with the local platelet-surface proximity (Figure 2A, top). To quantitatively characterize the spatiotemporal dynamics at the single platelet level, we manually cropped single images of platelets and analyzed them by measuring the pixel-wise difference in the IRM intensities between consecutive frames of the movie, imaged at temporal resolution of 5 seconds, $\Delta \mathrm{IRM}_{t}=\mathrm{IRM}_{\mathrm{t}}-$ $\mathrm{IRM}_{\mathrm{t}+1}$, which we termed the focal activity map. This analysis provided us with quantitative information and visualization regarding the local platelet dynamics: positive values (yellow) imply local attachment, and negative values (blue) imply local detachment, in relation to the previous time frame (Figure 2A bottom). The brighter object observed at time $=0$ was a characteristic feature of the IRM imaging for an unattached, but close to the surface object, and was consistently associated with a platelet appearing slightly above the IRM focal plane (Figure 2A top, arrowhead). This out-of-focus feature provides the first visual cue for a near-future platelet attaching and spreading. Small attachment regions could be observed upon platelet appearance, at $\Delta \mathrm{IRM} 0$ (Figure 2A bottom, $\mathrm{t}=0$ sec., arrow). These local attachment regions corresponded to the future appearance of the first filopodia touching the surface (Figure 2A, $\mathrm{t}=45$ seconds, arrowhead) and appeared in 18 of 20 platelets that we analyzed. This observation suggests that platelets sense the substrate from a $\mu \mathrm{m}$-scale distance by extending thin protrusions toward the substrate that will later develop into stable filopodia. The appearance of full filopodia was 

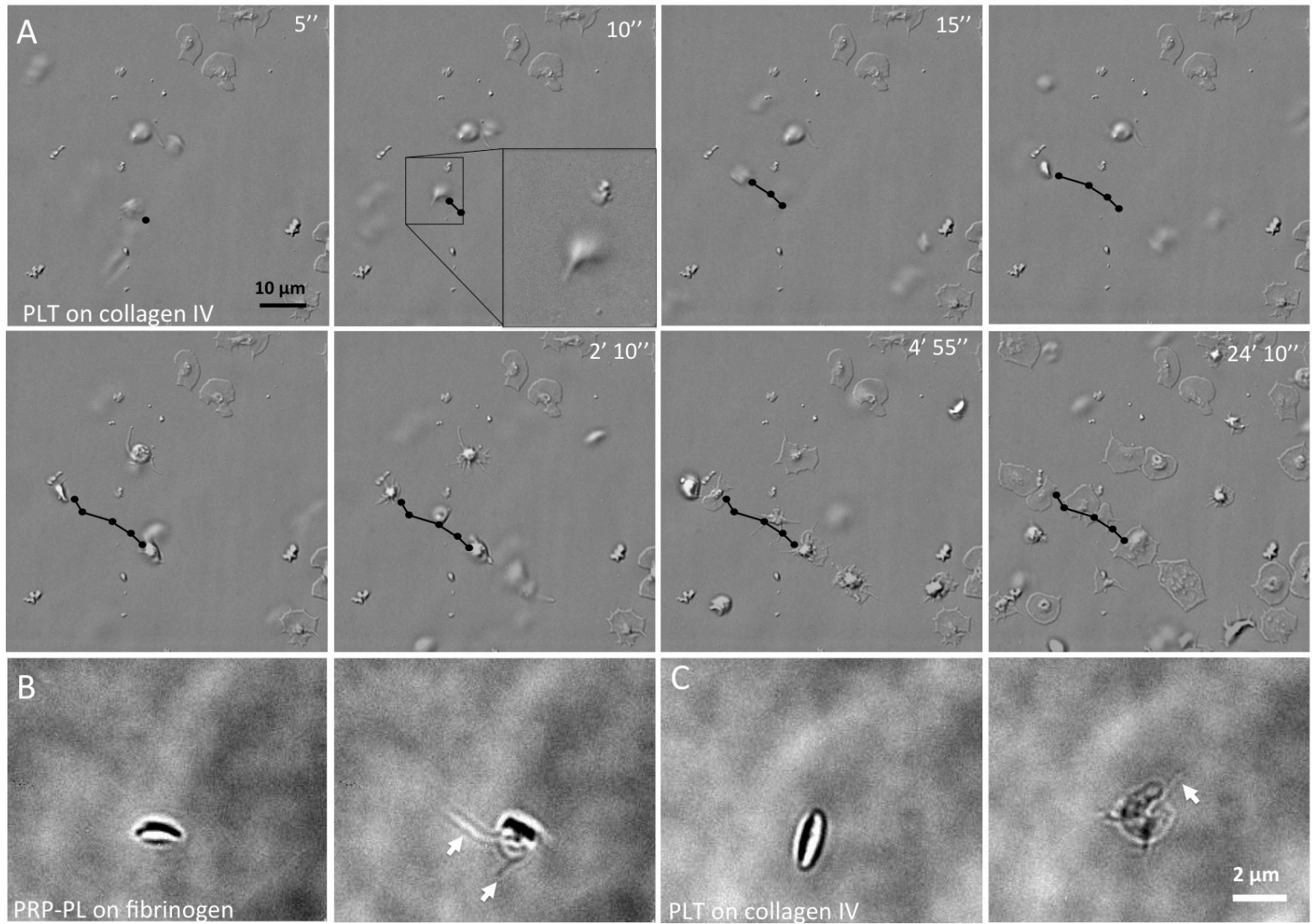

D

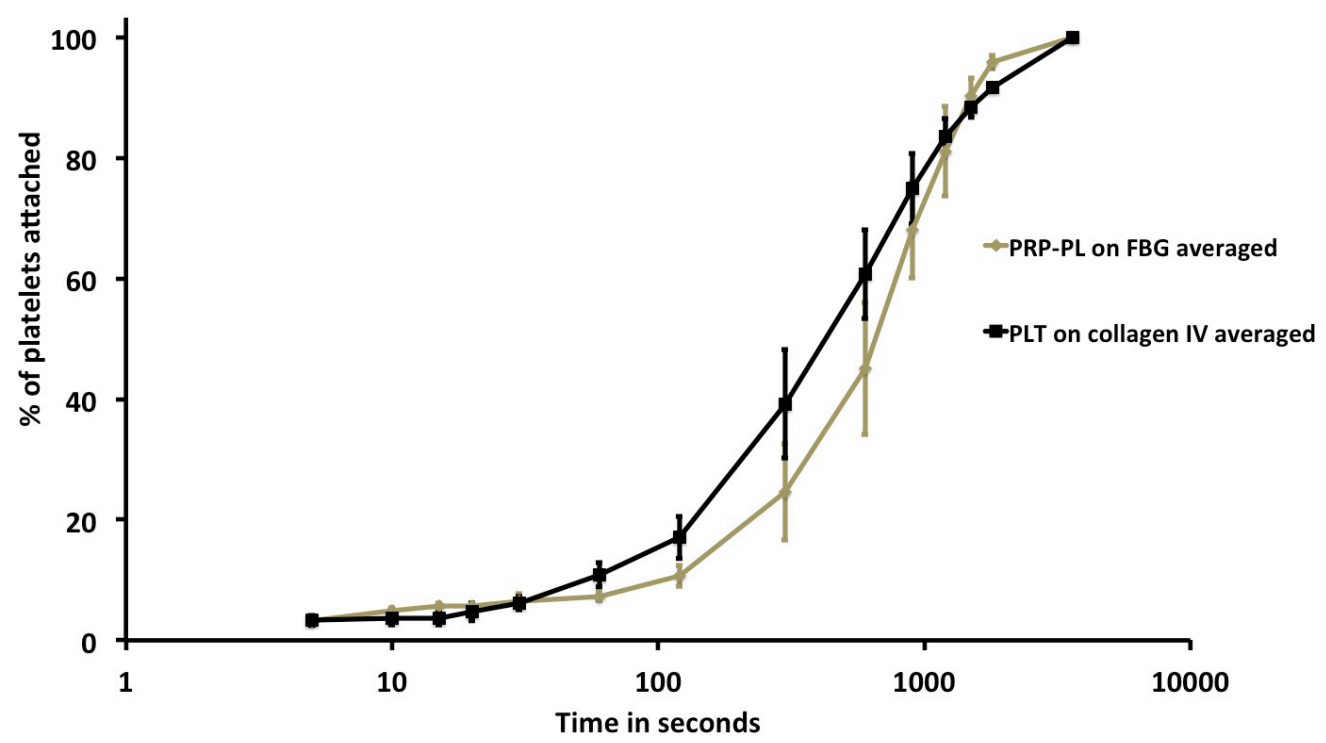

Figure 1. Observations of filopodia on platelets in solution on fibrinogen and collagen IV. (A) DIC, time-lapse microscopy of platelets attaching to collagen IV at different time points. Black arrow denotes the platelet in question. Scale bar: $10 \mu \mathrm{m}$. (B) Phase-contrast microscopy of PRP-PL on fibrinogen. Left: Discoid shape; Right: Platelet with filopodial extension. Scale bar: $5 \mu$ m. (C) Phase-contrast microscopy of PLT on collagen IV. Left: Discoid shape; Right: Platelet with filopodial extension. Scale bar: 5 Hm. (D) Platelet attachment to collagen IV or fibrinogen in percentage (\%) over time. Time in seconds, on a logarithmic scale. Platelets on collagen IV attach faster that on fibrinogen. Wilcoxon rank-sum test, $p$-value $<0.015$. N platelets: $F B G=121$, collagen IV=274. Error bars show Standard Error. 
A
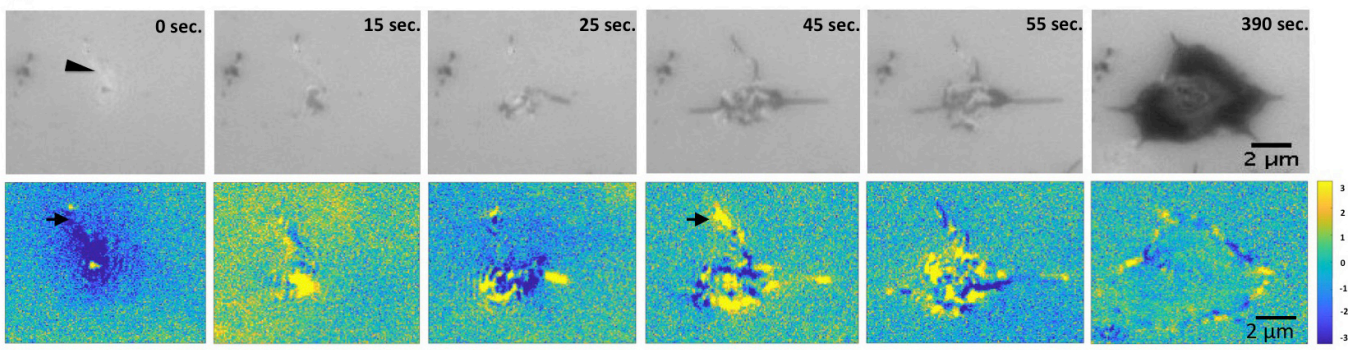

B

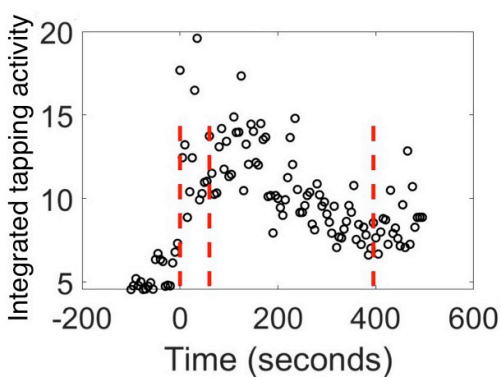

C

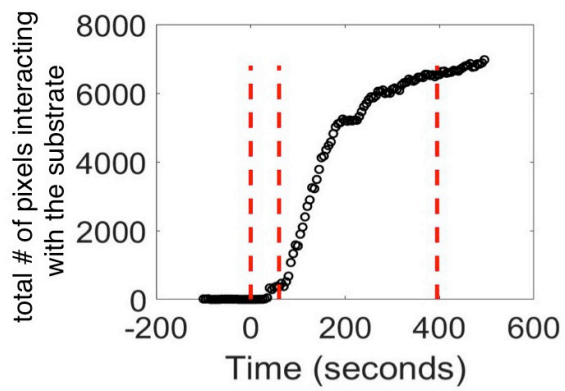

D
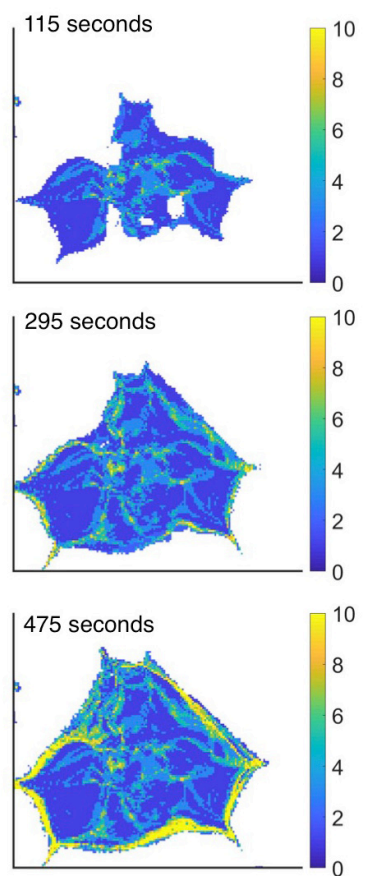
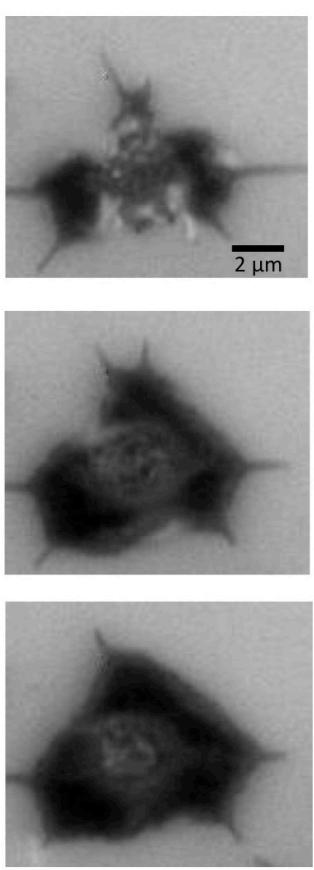

Figure 2. Quantification and image analysis of platelet spreading based on IRM live imaging for collagen IV. (A) Platelet spreading viewed by IRM, and the corresponding focal activity map, $\Delta I R M_{t}=I R M_{t}-I_{R M} M_{t+1}$. Positive values (yellow) imply local attachment; negative values (blue) imply local detachment (bottom right). One filopodia initially attaching and detaching (black arrow). Scale bar $2 \mu \mathrm{m}$ (B) Integrated tapping activity of platelets: the mean absolute value $|\Delta| \mathrm{RM} \mid$ at every time point. X-axis: Time in seconds. Y-axis: Platelet mean activity. Red dotted lines separate the phases: background, prior to platelet attachment, filopodial spreading phase, lamellipodial spreading phase, and the fully spread phase. (C) Total number of pixels interacting with the surface over time. Time in seconds. (D) Accumulated attachment and detachment over time shown by activity map, yellow means more attachment events, blue means fewer attachment event. Images on the right correspond IRM images. Scale bar 2 um.

characterized by local instability; some regions in the platelet were attaching to the substrate, while others were detaching concurrently, we termed this initial stage in platelet spreading, as the filopodial spreading stage (Figure $2 \mathrm{~A}, \mathrm{t}=45,55$ seconds). The filopodial stage was followed by a lamellipodial spreading stage, where local spatial instability was specifically observed at the platelet periphery, lamellipodia formed and platelet spreading preceded until reaching a fully spread morphology (Figure 2A, $\mathrm{t}=390$ seconds).

To examine the platelet spreading process in a more quantitative manner, we developed a custom pipeline to analyze the local attachment/detachment dynamics in IRM time-lapse and applied it to platelets spreading on collagen IV surfaces. Briefly, we overlaid a $3 \times 3$ pixel grid of bins on the $\Delta \mathrm{IRM}$ image, and collected temporal statistics on the attachment/detachment activity within these bins (see Methods). The overall attachment/ detachment activity of a platelet, which we call the platelet's integrated tapping activity, was defined as the mean absolute value $|\Delta I R M|$ at every time point (Figure $2 B$ ). This measure constituted the integration of all local alterations in the focal plane, encompassing both attachment and detachment events. A platelet's integrated tapping activity gradually increased during the filopodial stage, reaching a peak in activity, once this stage was complete. Platelet activity, then gradually decreased until it reached a plateau at the fully spread stage, 


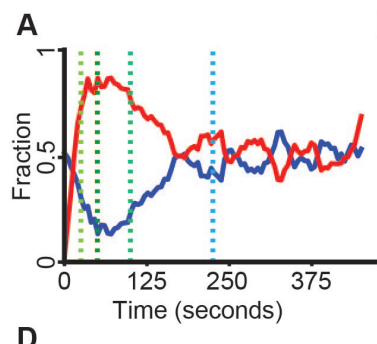

B
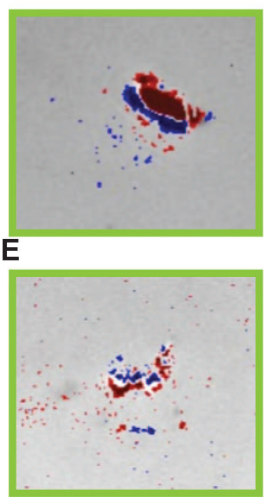
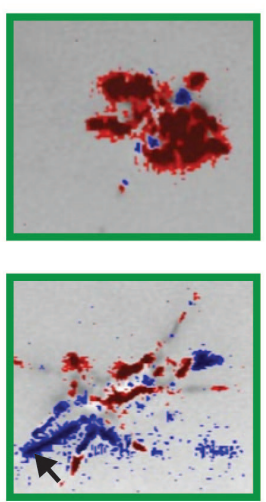
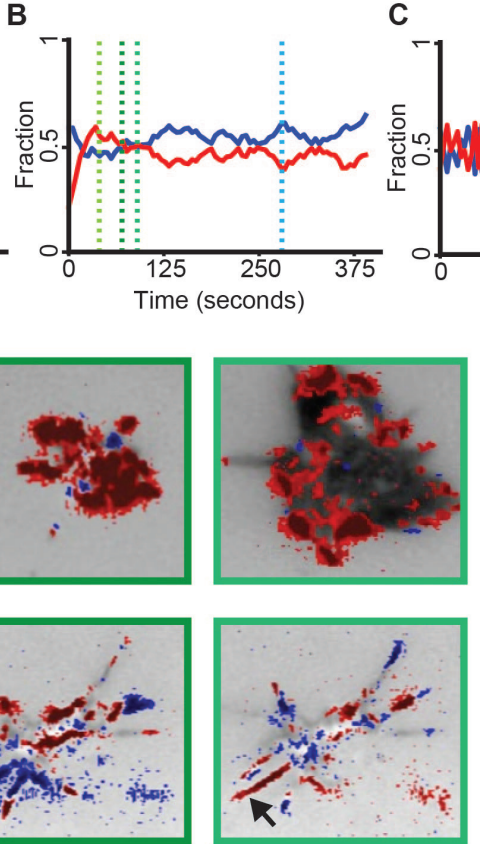

C

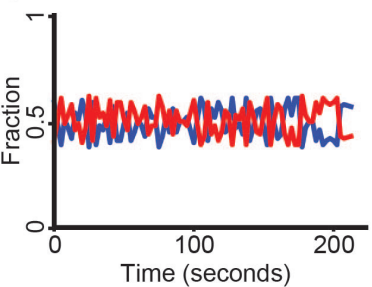

Figure 3. Differential platelet spreading: collagen IV versus fibrinogen. (A-C) Temporal profile of the fraction of attachment (red) versus detachment (blue) events during platelet spreading: (A) collagen-IV substrate, (B) fibrinogen substrate, (C) control - region with no platelet. (D and E) Spatiotemporal dynamics of attachment (red) versus detachment (blue) events during platelet spreading. Time corresponds to the vertical dashed line in panels A-B correspondingly: (D) Collagen-IV substrate. Platelet spreading is characterized by attachment, initially throughout the cell and later at the platelet periphery. (E) Fibrinogen substrate. Platelet spreading is characterized by the attachment and detachment of entire filopodia structures (arrow heads).

which was above the initial integrated tapping activity before the platelet appeared. This was found to be consistent for most of the platelets measured (Extended data: Figure $\mathrm{S}^{24}$ ).

We next used a classical threshold-based segmentation method to separate the pixels of each time point into foreground and background pixels based on their IRM intensities ${ }^{22}$ (see Methods). "Foreground" pixels had lower IRM intensities and were thus touching or very close to the surface, we termed these pixels as "interacting with the surface". The number of pixels interacting with the surface over time showed a slow increase during the filopodial stage, and increased rapidly during the lamellipodial stage until reaching the fully spread morphology (Figure 2C), this was consistent across several platelets (Extended data: Figure $\mathrm{S} 2^{24}$ ). By following the accumulated number of transitions from interaction to notinteracting with the surface at every pixel over time (see Methods), we observed that transitions occurred at the cell periphery as marked by no change in transitions accumulation in the platelet's interior over time (Figure 2D). The "vein"-like trails from the platelet center to its periphery suggest that the same filopodium is responsible for the repetitive interaction with the surface prior to the more stable lamellipodia (Figure 2D; Extended data: Movie $\mathrm{S1}^{24}$ ). Altogether, these data suggested that on collagen IV platelets extend filopodia that interact with the substrate by local attachment-detachment tapping events. This filopodia sensing coincides with a steady increase in lamellipodial area, which eventually leads to overall platelet spreading on collagen IV. We observed similar results for platelets spreading on fibrinogen (Extended data: Figure $\mathrm{S}^{24}$ ).

On one hand, filopodia appeared during platelet immobilization on both collagen IV and fibrinogen surfaces. On the other hand, platelet spreading is less effective on fibrinogen surfaces that were characterized by impaired transition to lamellipodial spreading. To pinpoint how local spatiotemporal dynamics lead to global differential platelet spreading we identified the subgroup of pixels that undergo significant attachment/detachment activity (see Methods). By plotting the fraction of the active pixels that undergo significant attachment (red) versus significant detachment (blue) we observed that platelet spreading on collagen IV was initially characterized by rapid and dominating attachment (Figure 3A). This is in contrast to the unstructured pattern that represents those platelets on fibrinogen that did not reach the lamellipodia-spreading phase (Figure $3 \mathrm{~B}$ ) or control regions with no platelets (Figure 3C). Overlaying these highly active regions on top of the IRM image revealed a striking differential pattern between platelet dynamics on collagen IV versus fibrinogen. While, platelets on collagen were characterized by attachment activity, initially throughout the cell body and later at the platelet periphery (Figure 3D; Extended data: Movie S2 ${ }^{24}$ ), platelets on fibrinogen were characterized by the attachment and detachment of entire filopodia structures (Figure 3E; Extended data: Movie $\mathrm{S}^{24}$ ). These results suggest that filopodia- 
to-lamellipodia transition is key for the effective spreading of platelets on collagen IV and less-efficient spreading on fibrinogen.

\section{Role of microtubules in platelets adhesion to immobilized collagen IV}

A key step in platelet-substrate interaction is the transition from filopodial-to-lamellipodial spreading, which displayed different dynamics on the two surfaces (delayed by 1-2 minutes on collagen IV). In an attempt to understand the mechanism underlying the timing of this transition, we explored the cytoskeletal state of the platelets, particularly their microtubule (MT) dynamics by tagging live platelets with SIR-tubulin, which allows real-time monitoring of MT. These experiments clearly indicated that the vast majority $(\sim 94 \%, \mathrm{n}=199)$ of platelets adhering to collagen IV commonly extend MTs towards the platelet periphery, where it interacts with and penetrates to a single, existing filopodium (Figure $4 \mathrm{~A}$ and $\mathrm{B}$, time point 175 seconds). The extension was not confined to a primary filopodia, suggesting that it is not involved in filopodia formation per se. Further analysis of the platelet spreading showed that the early-protruding lamellipodium usually extended from the location of the main MT-targeted filopodium (Figure 4C and E).

In platelets spreading on fibrinogen we did not observe microtubule extension into filopodia, suggesting that this phenomenon is collagen IV-specific. This observation, combined with the common failure of platelets adhering to fibrinogen, to undergo complete filopodial-to-lamellipodial switch, suggests that local microtubule extension to the platelet's periphery plays a key role in the transition to lamellipodial spreading (Figure 4F). This notion is further supported by experiments showing that treatment of PLT/collagen IV with nocodazole impaired lamellipodia formation (Figure 4G), in agreement with $^{25}$.

The MT-targeted filopodia from a random platelet revealed that microtubules entered the filopodia, but did not reach the tip of the filopodia as seen by cryo-electron tomography (Figure 5A and B). We observed an abundance of actin filaments (black arrows) in the filopodia, but only 1-2 microtubules that entered the filopodial core. Overall, we located MTs in $~ 58 \%$ of the filopodia we imaged (Figure 5C). From the $3 \mathrm{D}$ view we could appreciate the organized actin bundles at the tip of the filopodia, whilst more dispersed and organized in a network closer to the shaft (Figure 5D). We also observe a plentitude of trans-membrane, presumably including $\alpha \operatorname{IIb} \beta 3$ integrin, receptors (Figure 5E).

Differential receptor-mediated surface recognition of PRP-PL and PLT, and their effects on the attachment and spreading on collagen IV and fibrinogen

When we first examined the interactions of both PLT and PRP-PL, with each of the two surfaces, striking differences in adhesion dynamics was found (Figure 6A and B). In the experiments presented in the previous sections of this article we have primarily compared the adhesion and spreading dynamics of PLT on collagen IV to those of PRP-PL to fibrinogen. The primary reason for this pairing of platelet type (PLT and PRP-PL) and matrix type (collagen IV and fibrinogen), respectively, was that switching these platelets-matrix pairs resulted in poor-to-no adhesion and spreading. Specifically, PLT on collagen IV were characterized by little aggregation, adhesion followed by filopodial, and then lamellipodial spreading as described above (see also Figure 6A). PRP-PL, on the other hand, failed to attach and spread on collagen (Figure 6B).

This behavior was essentially mirror-imaged by PRP-PL, which attached to fibrinogen surfaces and proceeded by extending filopodia. Some of these platelets further developed lamellipodia and progressed to the fully spread state, while others remained in the filopodia state (Figure 6C). The attachment of PLT to fibrinogen was rather poor; only few platelets adhered to the matrix, and even fewer spread on it (Figure 6D).

The failure of PRP-PL to adhere to collagen IV was attributed to von Willebrand factor (VWF), which was present in the plasma, and effectively block collagen IV binding ${ }^{26,27}$. It was further shown that the addition of VWF to PLT preparations blocked the attachment and spreading (Figure 6E and F).

The limited ability of PLT to adhere and spread on fibrinogen is not entirely clear and could be attributed to the removal of VWF when the plasma is removed. VWF binding to integrin $\alpha \operatorname{IIb} \beta 3$ could play a role in this process $^{28}$.

The overall spreading rate of PLT on collagen IV, from initiation to maximal extension, was $\sim 2.5$-fold slower than that of PRP-PL on fibrinogen (10 minutes, compared to 25 minutes, Figure 7A and B). Platelets on collagen IV exhibited longer filopodia and lamellipodia spreading phases, than platelets on fibrinogen (Figure 7C). The filopodia and lamellipodia spreading phases on both surfaces were demonstrated by scanning electron microscopy (Figure 7D-I).

Beyond the distinct spreading dynamics of PLT and PRP-PL on collagen IV and fibrinogen, their "spreading endpoints" were different. Within $1 \mathrm{~h}$ after plating, $76 \%$ of PLT on collagen completed the filopodia-lamellipodia transition, while only 57\% PRP-PL on fibrinogen failed to go through the filopodial-lamellipodial switch (Figure 8A). To explore the possibility that this arrest is due to insufficient activation of the $\alpha \mathrm{IIb} \beta 3$-receptor in the PRP-PL, we allowed these platelets to spread on fibrinogen for 30 minutes and then added the platelet activation agonists; $\mathrm{Mn}^{2+}$ or thrombin for additional 30 minutes (Figure 8B). These experiments clearly indicated that integrin ( $\alpha \operatorname{IIb} \beta 3)$ activation drives lamellipodia formation to full spreading (Figure $8 \mathrm{~B}$ and $\mathrm{C}$ ). To test the differential role of integrin $\alpha \operatorname{IIb} \beta 3$ in the adhesion and spreading on fibrinogen and collagen IV, we seeded PLT and PRP-PL on collagen IV and fibrinogen in the presence or absence of the $\alpha \operatorname{IIb} \beta 3$ inhibitor sn528 $8^{29}$. This inhibitor had limited effect on the attachment and spreading on collagen while the adhesion to fibrinogen was completely blocked in line with earlier studies ${ }^{30}$ (Figure $9 \mathrm{~A}$ and B). 

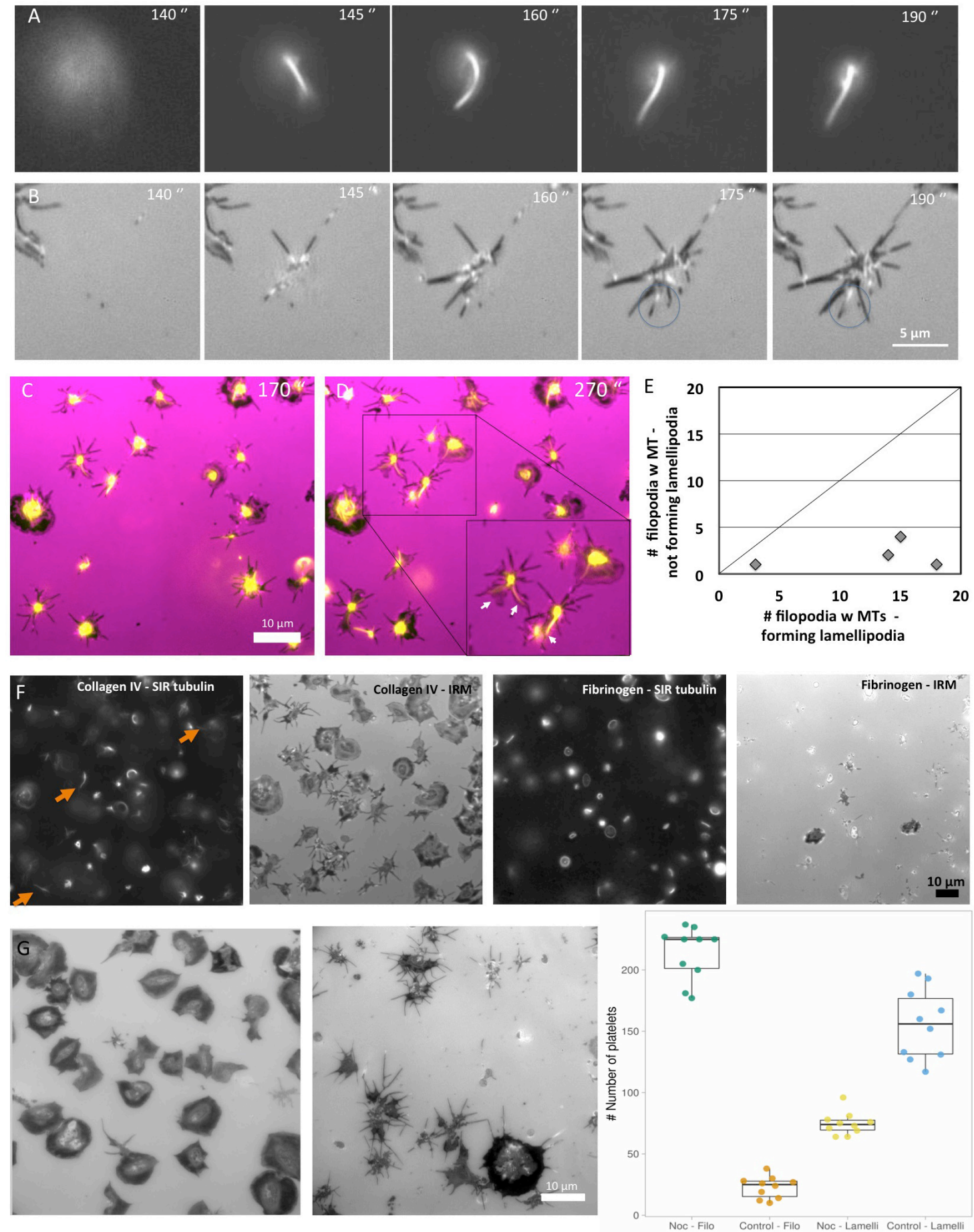

Figure 4. Platelet spreading and visualization of microtubule extensions. (A) Platelet spreading every 5 seconds, as viewed by fluorescence microscopy (FM). Platelets tagged with tubulin are pictured in the upper row (B) In the row below, platelets are imaged by IRM scale bar 5 um. (C) Platelet spreading on collagen IV at time point $170 \mathrm{sec}$. IRM (pink) overlay with SIR-tubulin (yellow). (D) Same as (C), at time point $270 \mathrm{sec}$. Lamellipodia formed where the SIR-tubulin extension is seen in filopodia (white arrows). (E) Scatter plot showing each experiment, how many platelet filopodia with microtubules extensions exhibit lamellipodia formation. X-axis number of MT containing filopodia forming lamellipodia, y-axis number of MT containing filopodia not forming lamellipodia. $N$ experiments $=4$ and $n$ platelets=58. (F) Washed platelets (PLT), on collagen IV and fibrinogen surfaces from left to right seen by SIR-tubulin and corresponding IRM, Sir-tubulin and corresponding IRM. Orange arrows indicate the dominant microtubule extension. Scale bar $10 \mu \mathrm{m}$. (G) IRM images from time-lapse movies of platelets treated with/without nocodazole. Scale bar: $10 \mu \mathrm{m}$. Time point: $60 \mathrm{~min}$. Far right: boxplot showing platelet specific shape - filopodia or lamellipodia spread, with and without nocodazole treatment. We saw that there was a difference between platelets treated with nocodazole compared to the control in terms of the number of filopodia spread platelets and lamellipodia spread platelets. Wilcoxon sum rank test was performed ${ }^{*} p$-value $<0.01, \mathrm{~N}$ experiments $=10, \mathrm{n}$ platelets $=4669$ 

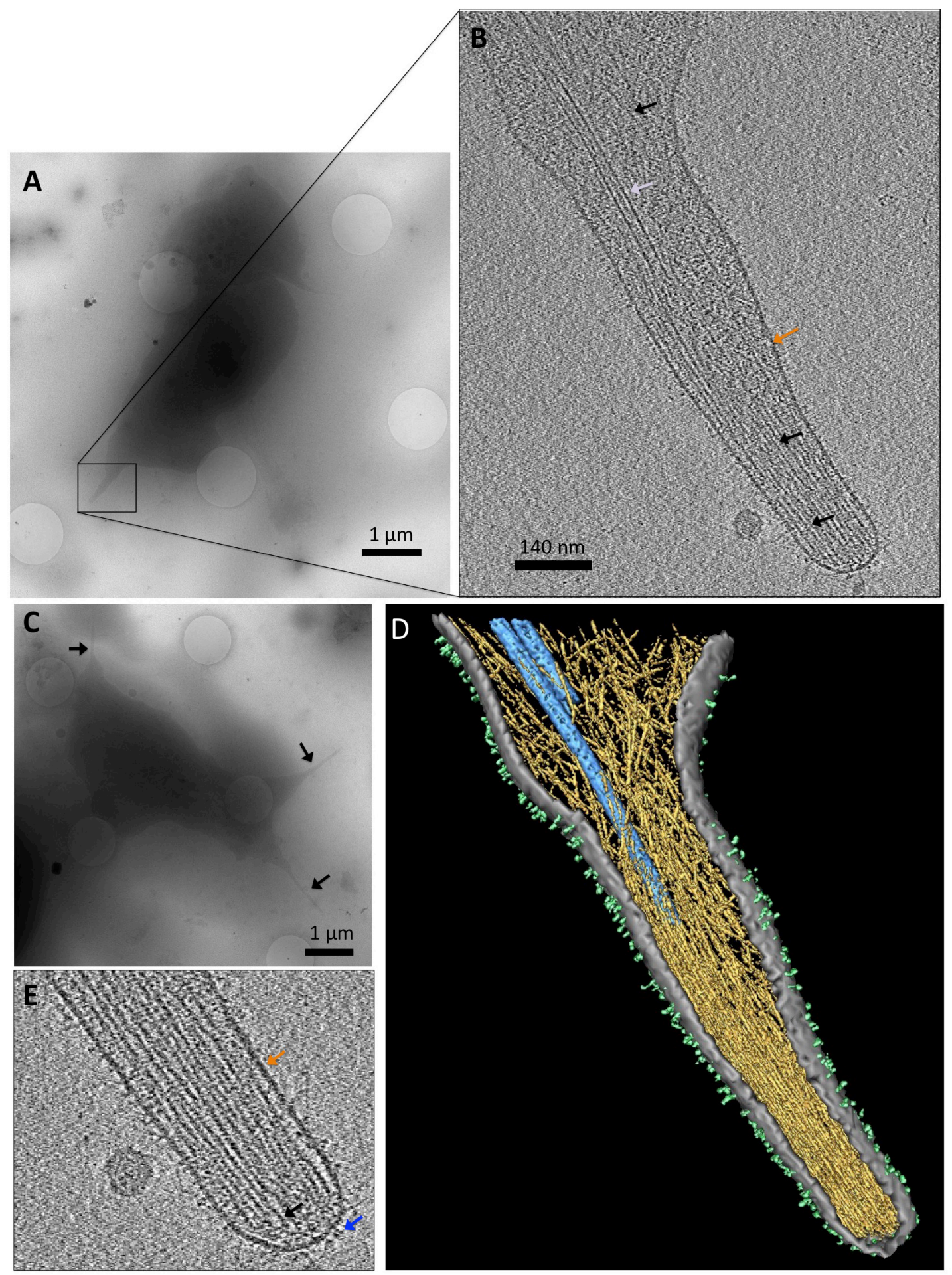

Figure 5. Cryo-electron tomography of a platelet filopodia on collagen IV. (A) Low magnification image of a whole platelet with filopodial protrusions. Scale bar: $1 \mu \mathrm{m}$. (B) The same platelet as in $(\mathbf{A})$, with a high magnification image of the filopodia in (A), black box. Slice from the 3D reconstructed volume. Visible is the membrane (orange arrow), microtubules (white arrow), and actin (black arrows). Scale bar: $140 \mathrm{~nm}$. (C) Low magnification image of a representative platelet with filopodial extensions (black arrows). Scale bar: $1 \mu \mathrm{m}$ (D) Rendered volume of the whole tomogram. Actin in yellow, microtubules in blue, membrane in grey, and surface receptors in light green. (E) Zoom-in view of (B), a slice from the 3D reconstructed volume. We are able to see the membrane (orange arrow), actin (black arrow), and surface receptors resembling integrins (blue arrow). 

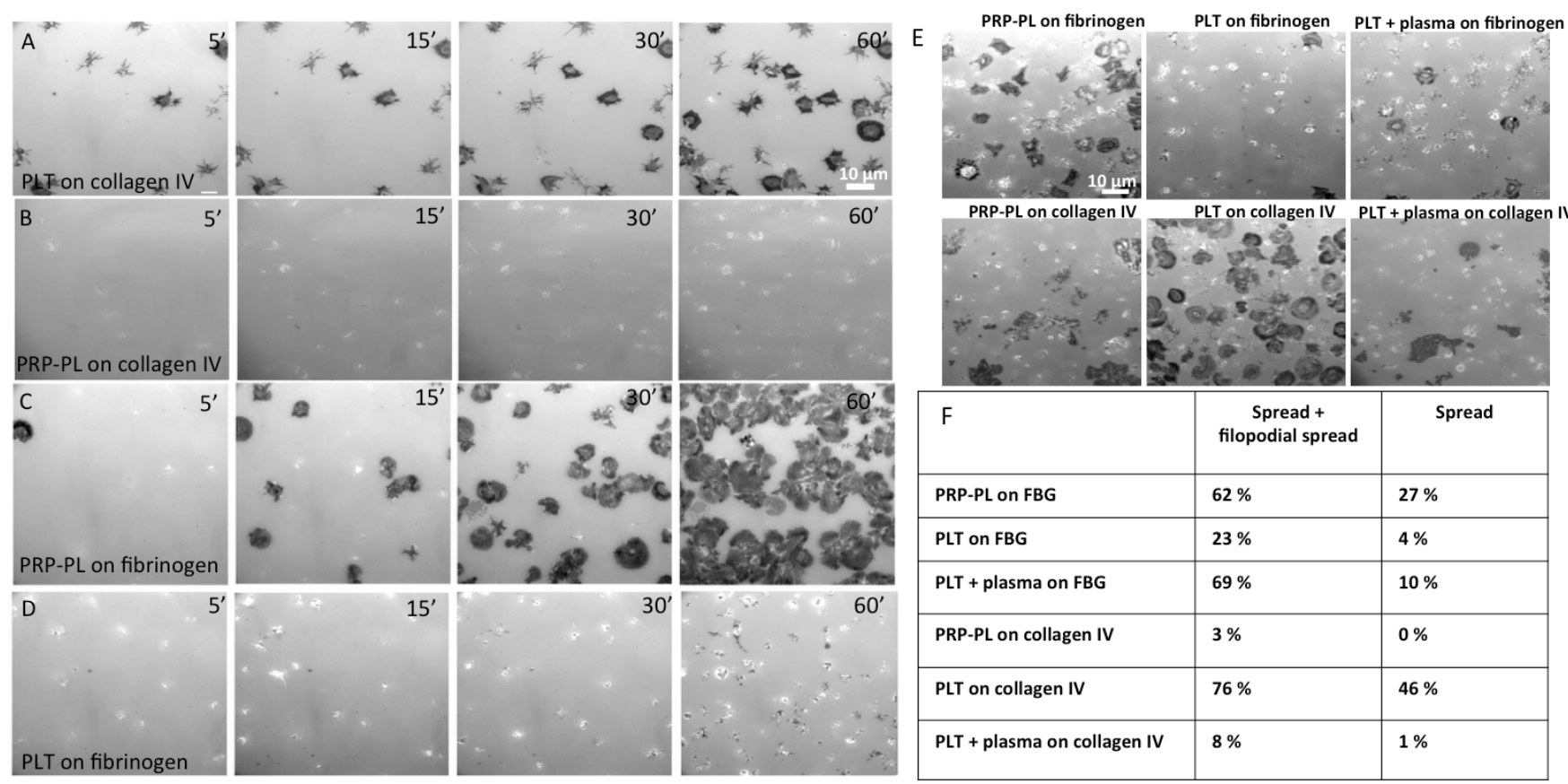

Figure 6. Platelet dynamics on fibrinogen and collagen IV surfaces. (A-E) IRM imaging of platelet spreading for 1 hour. Scale bar: $10 \mu \mathrm{m}$. (A) PLT spreading on collagen IV surfaces. (B) PRP-PL spreading on collagen IV. (C) PRP-PL spreading on fibrinogen. (D) PLT spreading on fibrinogen. (E) PRP-PL and PLT spreading on fibrinogen and collagen IV, with and without the addition of plasma to the PLT, 1 hour postplatelet seeding. (F) Percentage of platelets in each morphological state, 1 hour post-platelet seeding.

\section{Discussion}

Our spatiotemporal analysis of platelet spreading demonstrated that platelets sense their environment well before they firmly attach to the functionalized surfaces. During these early spreading stages, the platelets undergo structural changes, manifested by the extension of filopodia, through which intermittent interactions with the surface are established, attenuate the platelets' mobility, but do not block it (Figure 10A). These early adhesions, where the platelet changes from a discoid to a spherical shape, and after a short time $(<1 \mathrm{~min})$ extends actin-rich filopodia protrusions, followed by lamellipodia spreading was described in the literature, but their dynamic properties, and transition into stable adhesions were not characterized before ${ }^{20,31,32}$. Intermittent adhesions formed during the mobile phase (Figure 10B), led to immobilization of the adhering platelets, and triggered the subsequent spreading process. The dynamics of platelet spreading during the first minute after reaching the vicinity of the matrix was similar for platelets spreading on collagen or fibrinogen surfaces (Figure 10B, left from the "1 minute" time point). On the other hand, later events in the platelets' response appeared matrix-dependent. Filopodial spreading persisted for longer periods and the onset of lamellipodial spreading was delayed on collagen IV. An additional and significant difference is the microtubule extension event, which is apparent only in the cells spreading on collagen. Taken together, these results suggest that distinct environmental signaling cues, induced by collagen IV and fibrinogen, are responsible for the differential late ( 1-30 minutes) responses of the platelets.
To monitor the early transition of the intermittent adhesions into more stable ones, we have employed and combined realtime DIC and IRM microscopy and developed dedicated image analysis techniques. Our dynamic adhesion data analysis revealed a novel pattern of oscillations, during which the platelet first moves closer to the surface, then further away, then closer again, the so-called tapping events. The frequency of these fluctuations was in the order of $\sim 5-10$ seconds, and especially obvious at the filopodial tips. These observations show that early platelet attachment events are oscillatory in nature, and might be coordinated by the platelet itself, yet the mechanism underlying these adhesion oscillations is still unknown. These integrated tapping active events were restricted to the early filopodial spreading stage, leveled out over the course of platelet spreading, and decreased toward the end of the lamellipodial extension period.

Taken together these dynamic adhesion data suggest a model whereby platelets sense the surface through filopodial protrusions while still hovering over the surface (in a time-scale of a few-to-tens of seconds after reaching the surface vicinity). During this the platelet senses the substrate by intermittently touching it or 'tapping' on it, before they undergo extensive spreading (Figure 10A). We hypothesize that this 'tapping' process is a rather general mechanism for pre-adhesion sensing, that can play key role in focal complex or focal adhesion formation, as well as in the local organization of cytoskeletal components such as actin and microtubules. In this manner, 

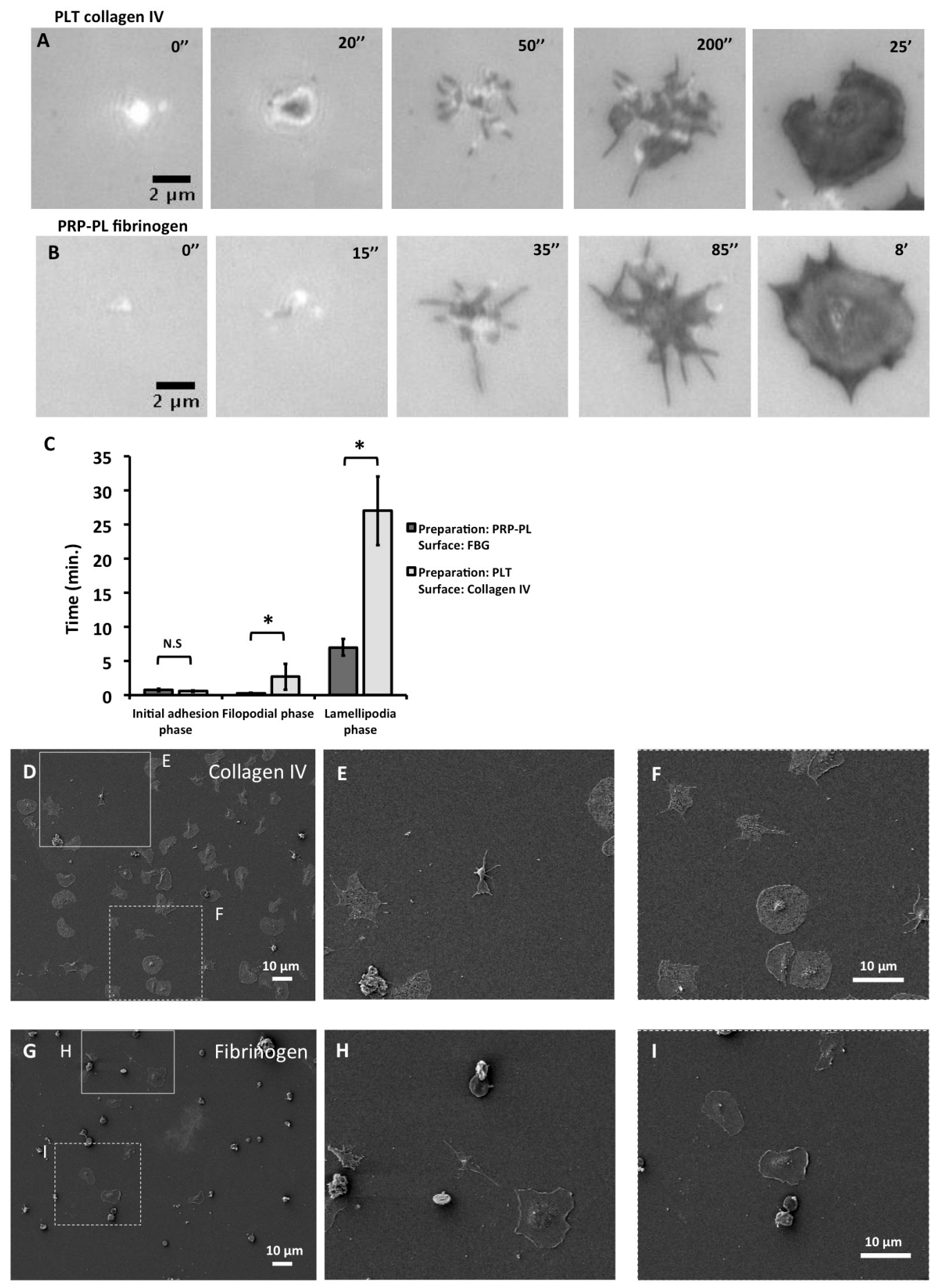

Figure 7. Use of IRM and SEM to image platelet dynamics on collagen IV and fibrinogen. (A) IRM (100X) of a platelet (PLT) spreading on collagen IV; (left to right) filopodia tapping and fluctuations (0", 50"), filopodia spreading (50", 200"), lamellipodial protrusion (200"), to the final, spread shape (25'). Scale bar: $10 \mu \mathrm{m}$. (B) IRM (100X) of a platelet (PRP-PL) spreading on fibrinogen; (left to right) filopodia tapping and fluctuations (0", 35"), filopodia spreading (35", 85"), lamellipodial protrusion (85") to the final, spread shape (8'). Scale bar: $10 \mu \mathrm{m}$. (C) Dynamics of platelet spreading. Graph showing the length of each of the phases described in (A) and (B). Wilcoxon rank sum test was used. * $\mathrm{p}$-value $<0.01, \mathrm{~N}$ platelets collagen IV=40, N platelets fibrinogen $=40$. Error bars show Standard Error. (D) SEM image (1.00 K X) of PLT on collagen IV. Scale bar: $10 \mu \mathrm{m}$. (E) SEM image $(1.00 \mathrm{~K} \mathrm{X)}$ of PLT on collagen IV, showing a filopodia spread platelet. (F) SEM image $(1.00 \mathrm{X} \mathrm{K})$ of PLT on collagen IV, showing a fully spread platelet $(\mathbf{G})$ SEM image (1.00 K X) of PRP-PL on fibrinogen. Scale bar: $10 \mu \mathrm{m}$. (H) SEM image $(1.00 \mathrm{~K} \mathrm{X})$ of PRP-PL on fibrinogen, showing a filopodia spread platelet. (I) SEM image (1.00 X K) of PRP-PL on fibrinogen, showing a fully spread platelet. Scale bar: $10 \mu \mathrm{m}$. 
A

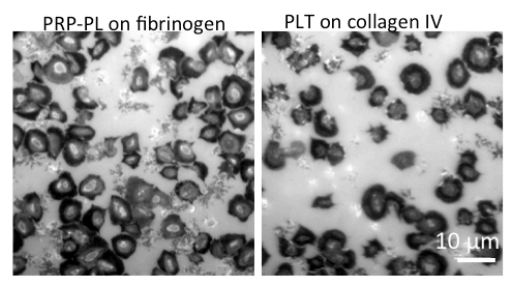

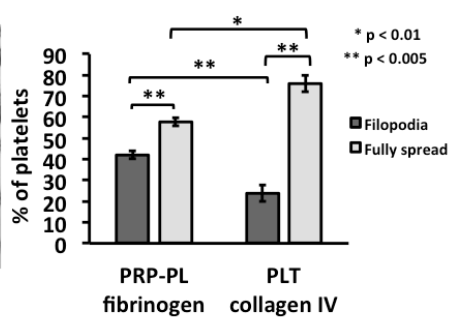

B

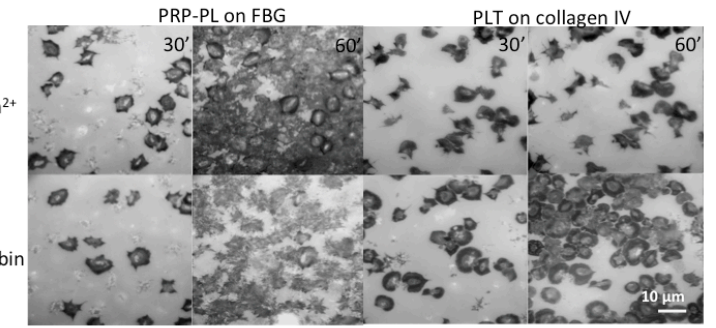

C
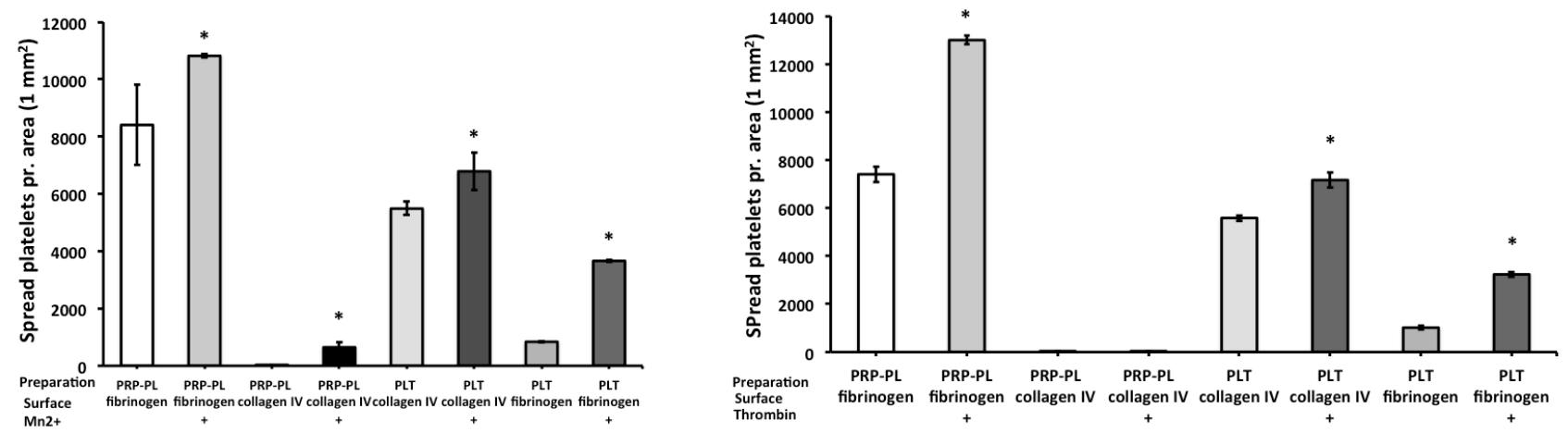

Figure 8. Platelet, PLT and PRP-PL adhesion to collagen IV and fibrinogen with and without $\mathbf{M n}^{2+}$ and thrombin agonists. (A) PRP-PL on fibrinogen, imaged by IRM (100X magnification). PLT on collagen IV, imaged by IRM (100X magnification). Scale bar: $10 \mu \mathrm{m}$. Right graph: \% distribution of filopodia and fully spread platelets. Platelet adhesion as a \% of filopodia spread platelets vs. fully spread platelets, between PLT on collagen IV and PRP-PL on fibrinogen. Scale bar: $10 \mu \mathrm{m}$. Wilcoxon rank-sum test was used, ${ }^{*} p$-value $<0.005$ and ${ }^{* *} p$-value $<0.01$. $\mathrm{N}$ experiments $=8$. $\mathrm{N}$ platelets: $\mathrm{FBG}=532$, collagen IV=542. Error bars show Standard Error. (B) Platelets on the surfaces at 30 min. and $60 \mathrm{~min}$. in the presence of manganese $\left(\mathrm{Mn}^{2+}\right)$ and thrombin. Scale bar: $10 \mu \mathrm{m}$. (C) Overview over how many spread platelets per area $\left(1 \mathrm{~mm}^{2}\right)$ of the platelet preparations: PLT and PRP-PL on fibrinogen and collagen IV, in the absence and presence of the two agonist: Left $\mathrm{Mn}^{2+}$ treatment, Right thrombin treatment. Wilcoxon rank-sum test was used, ${ }^{*} \mathrm{p}$-value $<0.05 . \mathrm{N}$ experiments $=10$. Error bars show Standard Error.

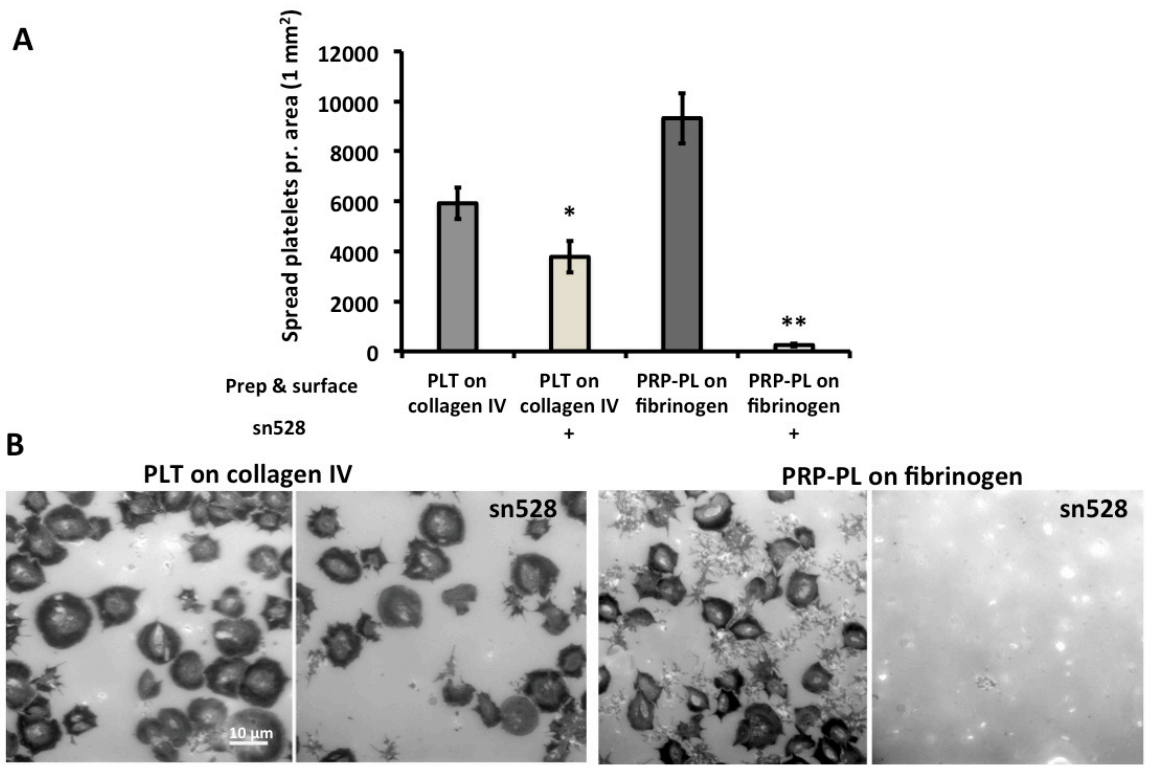

Figure 9. PLT and PRP-PL treated with or without the allb $\beta 3$ inhibitor sn528, on collagen IV and fibrinogen surfaces. (A) Inhibition of integrin $\alpha$ llb $\beta 3$ by sn528. Effect of integrin $\alpha$ llb $\beta 3$ inhibition shown by platelets on collagen IV and fibrinogen surfaces, with and without addition of the inhibitor sn528 (x-axis). Number of platelets per $1 \mathrm{~mm}^{2}$ : $y$-axis. Wilcoxon rank-sum test was used, ${ }^{*} \mathrm{p}$-value $<0.05$ and ${ }^{\star *} \mathrm{p}$-value $<0.001$. N experiments $=4$. Error bars show Standard Error. (B) PLT on collagen IV with and without the addition of the sn528 ligand. PRP-PL on fibrinogen with and without addition of the sn528 ligand. Scale bar: $10 \mu \mathrm{m}$. 

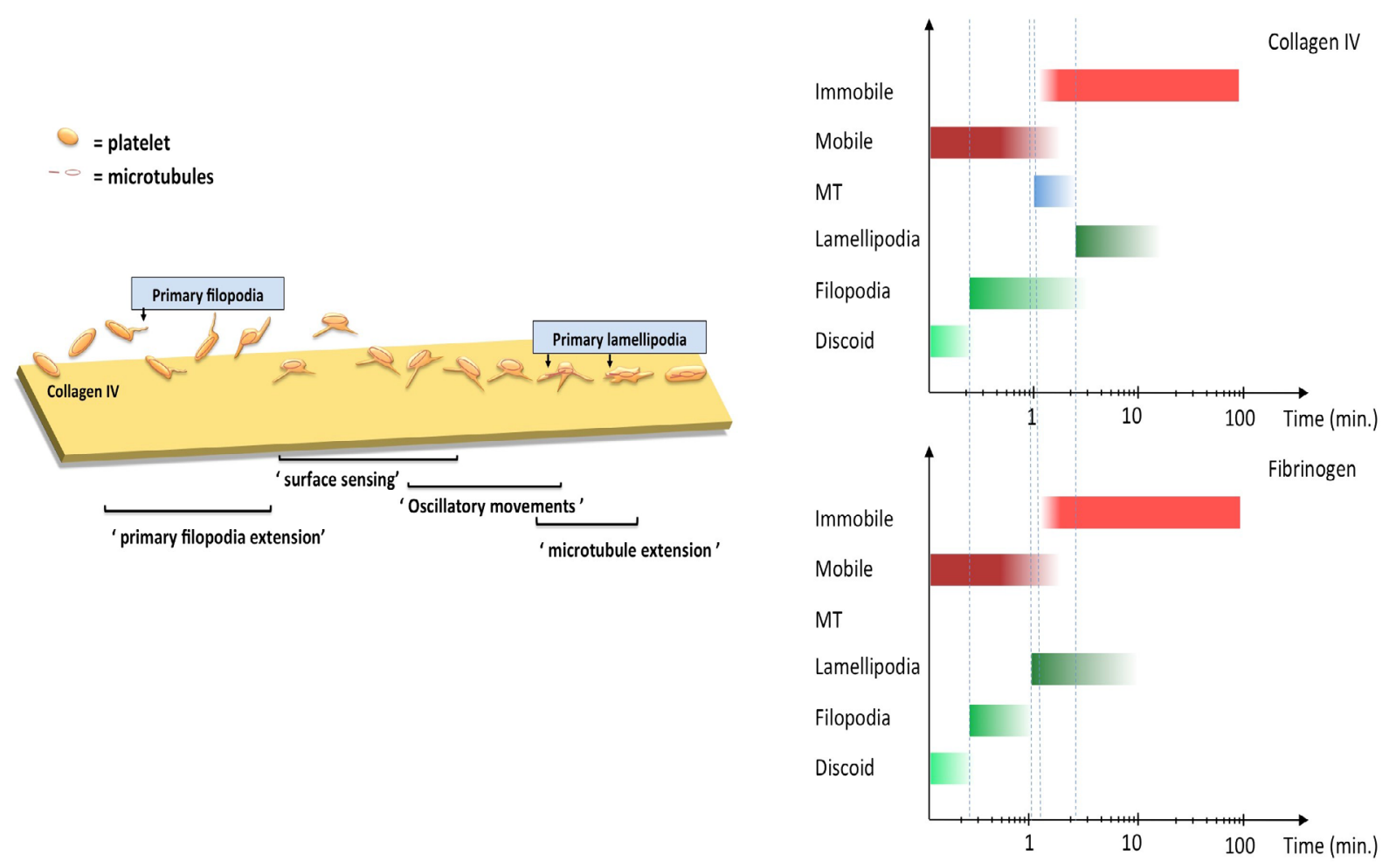

Figure 10. Suggested model for platelet early-stage adhesion. (A) The platelet (in orange) is floating as discoid shaped with the microtubule coil intact (red), it then extends filopodia while floating, hereby sensing the surface (yellow; collagen IV) and then exhibits oscillatory movements of attachment and detachment. It then attaches and sends out firmly attached protruding filopodia, one of them containing a dominant microtubule extension in the location where we see the primary lamellipodia forming and then continues to spread. (B) Overview of platelet temporal functional interplay. X-axis shows time, $\mathrm{y}$-axis shows a diverse range of parameters under temporal investigation. Red shades correspond to either a mobile or immobile platelet. Blue indicates microtubule polymerization and the shades of green are the different morphological shapes of the platelet phases: discoid, filopodia and lamellipodial. The gradient illustrates if the certain parameter has more or less over all activity.

the local sensing by filopodial protrusions may have a global effect, controlling platelet transformation from an early dynamic phase to a more mature and stable one.

One of the intriguing observations, reported here, is the apparent involvement of microtubules in the regulation of platelets' spreading on collagen IV. These findings suggest that microtubules play a role in the progression of lamellipodia extension, in line with Waterman-Storer's observation that microtubules are involved in actin-based protrusions at the leading-edge of migrating fibroblasts ${ }^{33}$. The absence of lamellipodial protrusions in most platelets adhering to fibrinogen might be attributed to the lack of microtubule-mediated stimulation. Previous studies demonstrated that the interplay between actin and microtubules is bidirectional, and can be directed by specific signaling pathways ${ }^{34}$. The functional significance of the microtubule extension and its relevance to the regulation of platelet spreading was reinforced by the demonstration that microtubule disruption by nocodazole blocked lamellipodia formation, in spreading PLTs.

When we examined PLT on collagen IV and PRP-PL on fibrinogen, we see that the results indicate striking differences in adhesion dynamics. The primary difference of the two systems is in the receptors mediating the adhesion. In platelets the attachment to collagen IV follow the 2-site 2-step model where the initial attachment and tethering is orchestrated by the GPVI receptor, followed by inside-out activation of integrin $\alpha 2 \beta 1^{35,36}$. The collagen receptor complex GPVI-FcR $\gamma$-chain mediates platelet activation byligand-mediated clustering of the receptor which triggers an increase in Src family kinases (SFKs) activity and downstream tyrosine phosphorylation of enzymes, adaptors, and cytoskeletal proteins that collectively propagate the signal and coordinate platelet activation in the sense of cytoskeletal remodeling including actin protrusions, degranulation and integrin activation ${ }^{37,38}$.

PRP-PL attached to fibrinogen presumably via integrin $\alpha \operatorname{IIb} \beta 3$, but also via GP-VI, as recent studies have shown ${ }^{39,40}$. Integrin $\alpha \operatorname{IIb} \beta 3$ can be activated by physiological agonists, such as ADP, thrombin or Manganese. When integrin $\alpha \operatorname{IIb} \beta 3$ is activated it transforms from a low- to high-affinity state. In this manner it binds fibrinogen and other ligands. When a ligand is bound, integrins cluster and the outside-in signaling cascade is stimulated. This drives essential platelet processes, such as attachment, spreading and thrombus formation. The exact 
mechanism involved in inside-out and outside-in signaling of integrin $\alpha \operatorname{IIb} \beta 3$ is not fully understood ${ }^{41,42}$. What we do know is that when integrin $\alpha \operatorname{IIb} \beta 3$ mediates outside-in signaling, it induces the tyrosine phosphorylation of multiple proteins. The SFKs plays a major role in these phosphorylation events. SFKs phosphorylate a host of signaling and cytoskeletal-associated proteins in platelets. This includes: phospholipase $\mathrm{C} \gamma 2$ (PLC $\gamma 2$ ), focal adhesion kinase (FAK), and adhesion- and degranulationpromoting adaptor protein (ADAP, also known as SLAP-130), which leads to their recruitment and/or activation ${ }^{42}$. The partial platelet spreading on collagen IV and especially fibrinogen, were caused by receptor inactivation, which was overcome by treatment with $\mathrm{Mn}^{2+}$ and thrombin. Indicating the necessity of in particular the $\alpha \operatorname{IIb} \beta 3$ integrin in the full platelet activation response.

It was clear to us that the adhesion to fibrinogen is strictly dependent on $\alpha \operatorname{IIb} \beta 3$, which we showed could be blocked by the specific inhibitor of this integrin, sn528 ${ }^{10}$. Addition of this inhibitor to PLT plated on collagen had only limited effect, suggesting that the adhesion in that system is mediated mainly by the generic collagen IV receptors in platelets GP-VI and $\alpha 2 \beta 1$. This is consistent with the findings of Thornber et al. were they defined the necessity of $\alpha \operatorname{IIb} \beta 3$ in lamellipodia progression for platelets on collagen related peptide, fibrinogen and thrombin ${ }^{30}$.

We believe the washed platelets between the surfaces cause differences in adhesion and spreading of PLT, affecting the microtubule filopodial extension, which may play a role in promoting the spreading for platelets on collagen IV. The lack of a MT extension may hinder lamellipodia spreading for PLT on fibrinogen. This would indicate a surface-specific receptor response and downstream signaling either via GP-VI and $\alpha 2 \beta 1$ for collagen IV or $\alpha \operatorname{IIb} \beta 3$ for fibrinogen.

\section{Conclusions}

This study demonstrates the extraordinary capacity of human platelets to differentially sense and respond to distinct physiological microenvironments - collagen IV and fibrinogen. We show that the adhesion and spreading on these surfaces is a highly complex process that combines selective receptor-mediated interactions, and the triggering of downstream cytoskeletal reorganization processes, which reinforce the platelet's interaction with the external surfaces via specific membrane protrusions - filipodia and lamellipodia. These spatially and temporally coordinated events take place in a time scale of seconds to minutes, and involve integrin $\alpha \operatorname{IIb} \beta 3$ activation, for platelets adhering to fibrinogen and microtubule-based activation of lamellipodia in platelets adhering to collagen IV.

\section{Data availability}

Underlying data

Zenodo: IRM raw data (video format) and dataset (csv) supporting platelet attachment to collagen IV or fibrinogen in percentage over time (related to Figure 1), https://doi.org/10.5281/ zenodo. $3774819^{43}$.
Zenodo: Raw data, temporal profiling for platelet spreading dynamics (related to Figure 3). https://doi.org/10.5281/ zenodo. $3774823^{44}$.

Zenodo: Raw data for microtubule extension IRM images (videos) and raw data set (csv) (related to Figure 4), https://doi. org/10.5281/zenodo. $3774827^{45}$.

Zenodo: Raw data (IRM videos) of Nocodazole experiments (videos) and raw dataset for statistical purposes (csv) (related to Figure 4), https://doi.org/10.5281/zenodo.377483546.

Zenodo: Nocodazole experiment low mag images, IRM, raw data. Platelets fixed, imaged by IRM in low magnification for counting purposes. Platelets are either control or treated with nocodazole, https://doi.org/10.5281/zenodo.3774843 ${ }^{47}$.

Zenodo: Raw data to support percentage of platelets in each morphological state, 1 hour post-platelet seeding (related to Figure 8), https://doi.org/10.5281/zenodo.3774845 ${ }^{48}$.

Zenodo: Dynamics of platelet spreading over time with/without treatments with manganese and thrombin (related to Figure 8). Raw images of platelets treated with and without Manganese and thrombin (tif, jpegs) and raw data set (csv), https://doi.org/ 10.5281 /zenodo. $3774849^{49}$.

Zenodo: Un-cropped and unedited images/movies for all (DIC, movies, cryo-ET, SEM images). https://doi.org/10.5281/ zenodo. $3773437^{50}$.

\section{Extended data}

Figshare: Differential dynamics of early stages of platelet adhesion and spreading on collagen IV- and fibrinogen-coated surfaces, https://doi.org/10.6084/m9.figshare.c.494473824.

This project contains the following extended data:

- Figure S1. Platelet integrated activity. Integrated activity of platelets: the mean absolute value $|\Delta I R M|$ at every time point. X-axis: Time in seconds. Y-axis: Platelet mean activity. Red dotted lines separate the phases: background, prior to platelet attachment, filopodial spreading phase, lamellipodial spreading phase, and the fully spread phase.

- Figure S2. Interactions with the surface for collagen IV and fibrinogen. The number of pixels interacting with the surface over time for the surfaces collagen IV and fibrinogen. Time in seconds.

- Figure S3. Quantification and image analysis of platelet spreading, based on IRM live imaging for fibrinogen. (A) Platelet spreading viewed by IRM, and the corresponding focal activity map, $\Delta \mathrm{IRM}_{t}=\mathrm{IRM}_{\mathrm{t}}-\mathrm{IRM}_{\mathrm{t}+1}$. Positive values (yellow) imply local attachment; negative values (blue) imply local detachment (bottom right). One filopodia initially attaching and detaching (black 
arrow). Scale bar $2 \mu \mathrm{m}$ (B) Integrated tapping activity of platelets: the mean absolute value $|\Delta \mathrm{IRM}|$ at every time point. X-axis: Time in seconds. Y-axis: Platelet mean activity. Red dotted lines separate the phases: background, prior to platelet attachment, filopodial spreading phase, lamellipodial spreading phase, and the fully spread phase. (C) Total number of pixels interacting with the surface over time. Time in seconds. (D) Accumulated attachment and detachment over time shown by activity map, yellow means more attachment events, blue means fewer attachment event. Right images, correspond IRM images. Scale bar 2 um.

- Movie S1. Shows the accumulated number of transitions from interaction to not interacting with the surface at every pixel over time.
- Movie S2. Shows an overlay of the highly active regions on top of the IRM images over time on collagen IV.

- Movie S3. Shows an overlay of the highly active regions on top of the IRM images over time on fibrinogen.

Data are available under the terms of the Creative Commons Attribution 4.0 International license (CC-BY 4.0).

\section{Software availability}

IRM spreading dynamics source code available from: https:// github.com/assafZaritskyLab/IRM-Spreading-Dynamics

Archived source code as at time of publication: https://doi. org/10.5281/zenodo. $3770506^{21}$

License: GNU General Public License v3.0
1. Ruggeri ZM: Platelets in atherothrombosis. Nat Med. 2002; 8(11): 1227-1234. PubMed Abstract | Publisher Full Text

2. Varga-Szabo D, Pleines I, Nieswandt B: Cell adhesion mechanisms in platelets. Arterioscler Thromb Vasc Biol. 2008; 28(3): 403-412.

PubMed Abstract | Publisher Full Text

3. Cabodi $\mathrm{S}$, Di Stefano $\mathrm{P}$, Leal Mdel $\mathrm{P}$, et al.: Integrins and signal transduction. Adv Exp Med Biol. 2010; 674: 43-54.

PubMed Abstract | Publisher Full Text

4. Ruggeri ZM: Platelet adhesion under flow. Microcirculation. 2009; 16(1): 58-83 PubMed Abstract | Publisher Full Text | Free Full Text

5. Zucker MB, Nachmias VT: Platelet activation. Arteriosclerosis. 1985; 5(1): 2-18. PubMed Abstract | Publisher Full Text

6. Ruggeri ZM, Mendolicchio GL: Adhesion mechanisms in platelet function. Circ Res. 2007; 100(12): 1673-1685.

PubMed Abstract | Publisher Full Text

7. Frenette PS, Denis CV, Weiss L, et al.: P-Selectin glycoprotein ligand 1 (PSGL-1) is expressed on platelets and can mediate platelet-endothelial interactions in vivo. J Exp Med. 2000; 191(8): 1413-1422.

PubMed Abstract | Publisher Full Text | Free Full Text

8. Machlus KR, Italiano JE Jr: The incredible journey: From megakaryocyte development to platelet formation. J Cell Biol. 2013; 201(6): 785-796. PubMed Abstract | Publisher Full Text | Free Full Text

9. Thon JN, Italiano JE: Platelets: production, morphology and ultrastructure. Handb Exp Pharmacol. 2012; (210): 3-22. PubMed Abstract | Publisher Full Text

10. Zarka R, Horev MB, Volberg T, et al.: Differential Modulation of Platelet Adhesion and Spreading by Adhesive Ligand Density. Nano Lett. 2019; 19(3): 1418-1427.

PubMed Abstract | Publisher Full Text | Free Full Text

11. Jirousková M, Jaiswal JK, Coller BS: Ligand density dramatically affects integrin alpha Ilb beta 3-mediated platelet signaling and spreading. Blood. 2007; 109(12): 5260-5269.

PubMed Abstract | Publisher Full Text | Free Full Text

12. Vlodavsky I, Eldor A, HyAm E, et al:: Platelet interaction with the extracellular matrix produced by cultured endothelial cells: a model to study the thrombogenicity of isolated subendothelial basal lamina. Thromb Res. 1982; 28(2): 179-191.

PubMed Abstract | Publisher Full Text

13. Lickert S, Sorrentino S, Studt JD, et al:: Morphometric analysis of spread platelets identifies integrin $\alpha_{11 b} \beta_{3}$-specific contractile phenotype. Sci Rep. 2018, 8(1): 5428

PubMed Abstract | Publisher Full Text | Free Full Text

14. Lee $\mathrm{D}$. Fong KP. King MR et al: Differential dynamics of platelet contact and spreading. Biophys J. 2012; 102(3): 472-482.

PubMed Abstract | Publisher Full Text | Free Full Text

15. Haydon GB, Taylor DA: Microtubules in hamster platelets. J Cell Biol. 1965; 26(2): 673-676.

PubMed Abstract | Publisher Full Text | Free Full Text
16. Behnke O: Further studies on microtubules. A marginal bundle in human and rat thrombocytes. J Ultrastruct Res. 1965; 13(5): 469-477. PubMed Abstract | Publisher Full Text

17. Italiano JE Jr, Richardson JL, Patel-Hett S, et al:: Angiogenesis is regulated by a novel mechanism: pro- and antiangiogenic proteins are organized into separate platelet alpha granules and differentially released. Blood. 2008; 111(3): 1227-1233.

PubMed Abstract | Publisher Full Text | Free Full Text

18. White JG: Fine structural alterations induced in platelets by adenosine diphosphate. Blood. 1968; 31(5): 604-622.

PubMed Abstract | Publisher Full text

19. Hartwig $\mathrm{JH}$ : Mechanisms of actin rearrangements mediating platelet activation. J Cell Biol. 1992; 118(6): 1421-1442.

PubMed Abstract | Publisher Full Text | Free Full Text

20. Bearer EL, Prakash JM, Li Z: Actin dynamics in platelets. Int Rev Cytol. 2002; 217: 137-182. PubMed Abstract | Publisher Full Text | Free Full Text

21. assafZaritskyLab: assafZaritskyLab/IRM-Spreading-Dynamics: IRM-SpreadingDynamics-BGU-ReleaseV1.0 (Version v1.0.0). Zenodo. 2020. http://www.doi.org/10.5281/zenodo.3770506

22. Otsu N: A Threshold Selection Method from Gray-Level Histograms. IEEE T Syst Man Cy. 1979; 9(1): 62-66. Publisher Full Text

23. Nickell S, Förster F, Linaroudis A, et al:: TOM software toolbox: acquisition and analysis for electron tomography. J Struct Biol. 2005; 149(3): 227-234. PubMed Abstract | Publisher Full Text

24. Horev M: Supplementary data. figshare. 2020 http://www.doi.org/10.6084/m9.figshare.c.4944738.v1

25. Vasquez RJ, Howell B, Yvon AM, et al:: Nanomolar concentrations of nocodazole alter microtubule dynamic instability in vivo and in vitro. $\mathrm{Mol} \mathrm{BiO}$ Cell. 1997; 8(6): 973-985. PubMed Abstract | Publisher Full Text | Free Full Text

26. Flood VH, Schlauderaff AC, Haberichter SL, et al:: Crucial role for the VWF A1 domain in binding to type IV collagen. Blood. 2015; 125(14): 2297-2304. PubMed Abstract | Publisher Full Text | Free Full Text

27. Fuchs B, Budde U, Schulz A, et al.: Flow-based measurements of von Willebrand factor (VWF) function: binding to collagen and platelet adhesion under physiological shear rate. Thromb Res. 2010; 125(3): 239-245. PubMed Abstract | Publisher Full Text

28. Ozaki $\mathrm{Y}$, Suzuki-Inoue $\mathrm{K}$, Inoue O: Platelet receptors activated via mulitmerization: glycoprotein VI, GPIb-IX-V, and CLEC-2. J Thromb Haemost. 2013; 11(Suppl 1): 330-339.

PubMed Abstract | Publisher Full Text

29. Weiss M, Frohnmayer JP, Benk LT, et al:: Sequential bottom-up assembly of mechanically stabilized synthetic cells by microfluidics. Nat Mater. 2018; 17(1): 89-96.

PubMed Abstract | Publisher Full Text

30. Thornber K, McCarty OJ, Watson SP, et al.: Distinct but critical roles for integrin alphallbbeta3 in platelet lamellipodia formation on fibrinogen, collagen-related 
peptide and thrombin. FEBS J. 2006; 273(22): 5032-5043. PubMed Abstract | Publisher Full Text

31. Mattila PK, Lappalainen P: Filopodia: molecular architecture and cellular functions. Nat Rev Mol Cell Biol. 2008; 9(6): 446-454.

PubMed Abstract | Publisher Full Text

32. Sandmann R, Koster S: Topographic Cues Reveal Two Distinct Spreading Mechanisms in Blood Platelets. Sci Rep. 2016; 6: 22357. PubMed Abstract | Publisher Full Text | Free Full Text

33. Waterman-Storer CM, Worthylake RA, Liu BP, et al.: Microtubule growth activates Rac1 to promote lamellipodial protrusion in fibroblasts. Nat Cell Biol. 1999; 1(1): 45-50.

PubMed Abstract | Publisher Full Text

34. Etienne-Manneville S: Actin and microtubules in cell motility: which one is in control? Traffic. 2004; 5(7): 470-477. PubMed Abstract | Publisher Full Text

35. Nieswandt B, Brakebusch C, Bergmeier W, et al.: Glycoprotein VI but not alpha2beta1 integrin is essential for platelet interaction with collagen. $E M B O \mathrm{~J}$. 2001; 20(9): 2120-2130.

PubMed Abstract | Publisher Full Text | Free Full Text

36. Nieswandt $B$, Watson SP: Platelet-collagen interaction: is GPVI the central receptor? Blood. 2003; 102(2): 449-461. PubMed Abstract | Publisher Full Text

37. Senis YA, Mazharian A, Mori J: Src family kinases: at the forefront of platelet activation. Blood. 2014; 124(13): 2013-2024.

PubMed Abstract | Publisher Full Text | Free Full Text

38. Inoue O, Suzuki-Inoue K, Dean WL, et al.: Integrin alpha2beta1 mediates outside-in regulation of platelet spreading on collagen through activation of Src kinases and PLCgamma2. J Cell Biol. 2003; 160(5): 769-780. PubMed Abstract | Publisher Full Text | Free Full Text

39. Mangin PH, Onselaer MB, Receveur N, et al:: Immobilized fibrinogen activates human platelets through glycoprotein VI. Haematologica. 2018; 103(5): 898-907.

PubMed Abstract | Publisher Full Text | Free Full Text

40. Savage B, Ruggeri ZM: Selective recognition of adhesive sites in surface-bound fibrinogen by glycoprotein Ilb-Illa on nonactivated platelets. J Biol Chem. 1991; 266(17): 11227-11233. PubMed Abstract

41. Bennett JS, Berger BW, Billings PC: The structure and function of platelet integrins. J Thromb Haemost. 2009; 7(Suppl 1): 200-205.

PubMed Abstract | Publisher Full Tex

42. Durrant TN, van den Bosch MT, Hers I: Integrin $\alpha_{11} \beta_{3}$ outside-in signaling. Blood 2017; 130(14): 1607-1619.

PubMed Abstract | Publisher Full Text | Free Full Text

43. Horev M: PhD. Zenodo. 2020. http://www.doi.org/10.5281/zenodo.3774819

44. Horev MB: PhD [Data set]. Zenodo. 2020. http://www.doi.org/10.5281/zenodo.3774823

45. Horev MB: PhD. Zenodo. 2020. http://www.doi.org/10.5281/zenodo.3774827

46. Horev MB: PhD. Zenodo. 2020 http://www.doi.org/10.5281/zenodo.3774835

47. Horev MB: PhD. Zenodo. 2020. http://www.doi.org/10.5281/zenodo.3774843

48. Horev MB: PhD. Zenodo. 2020 http://www.doi.org/10.5281/zenodo.3774845

49. Horev MB: PhD. Zenodo. 2020. http://www.doi.org/10.5281/zenodo.3774849

50. Horev MB: PhD. Zenodo. 2020. http://www.doi.org/10.5281/zenodo.3773437 


\section{Open Peer Review}

\section{Current Peer Review Status:}

\section{Version 1}

Reviewer Report 10 June 2020

https://doi.org/10.5256/f1000research.26040.r64006

(c) 2020 Schwarz U. This is an open access peer review report distributed under the terms of the Creative Commons Attribution License, which permits unrestricted use, distribution, and reproduction in any medium, provided the original work is properly cited.

\section{Ulrich Schwarz}

Institute for Theoretical Physics, Heidelberg University, Heidelberg, Germany

The authors have performed a quantitative analysis of platelet spreading on either collagen or fibrinogen coated surfaces. The battery of methods applied is pretty impressive: DIC, RICM (also called IRM), FM (with SIR-tubulin), cryoEM, SEM and quantitative image processing. Together these measurements provide interesting new insight into an important part of wound healing. In particular, this study reveals a surprisingly large role of microtubules, which are known to be important for platelet biology due to the marginal band, but here are also shown to insert into actin-based filopodia, which probe the surface in a "tapping" phase and later initiate lamellipodia. This study also shows the important role of different integrins selected by different matrix components (or blocked by plasma components like the VWF), leading to more complete spreading on collagen. All in all this study is an important advance for a quantitative understanding of the platelet spreading part of wound healing.

There are a few issues that should be addressed in a revision:

1. Platelet activation: the time point cero for the start of spreading seems to be defined by contact with the matrix-decorated surface. Later in the study additional activation by thrombin is mentioned. However, usually platelets are activated by thrombin or ADP at the beginning, and with variable doses. It should be explained why the study has been performed without such design (or if the reviewer misunderstood this part).

2. Relation to marginal band (MB): the new data on the microtubules is intriguing, but the relation to the marginal band is not clear. It is mentioned that the microtubules inserted into the filopodia arise from the MB. Can this be explained in more detail? Is this process regulated, e.g. by changing motor activity in the MB? It is well known that the MB coils after activation (compare e.g. Diagouraga et al. J Cell Biol 204: 177-185, 2014 ${ }^{1}$ ) and I wonder if this can be seen in Fig. 4.

3. Role of actomyosin: coiling of the MB is related to cortical contractility (compare above reference). Moreover it is well known that platelets also form stress fibers (compare Tanaka and Itoh, J Struct Biol 124: 13-41, 1998²) and recently it has been shown with traction force 
microscopy that they build up actomyosin-based traction forces with a time delay in regard to area spreading (Hanke et al. Soft Matter 14:6571-6581, 20183). For the general understanding of how the different processes are orchestrated during platelet spreading (as summarized in Fig. 10), it would be helpful to also explain the role of actomyosin contractility.

4. Comparison collagen versus fibrinogen: for most of the study these two conditions are treated symmetrically, but important Fig. 2 only shows data for collagen. I suggest to show the same analysis also for fibrinogen, if possible. This way it would also fit better to Fig. 3 .

\section{References}

1. Diagouraga B, Grichine A, Fertin A, Wang J, et al.: Motor-driven marginal band coiling promotes cell shape change during platelet activation.J Cell Biol. 2014; 204 (2): 177-85 PubMed Abstract I Publisher Full Text

2. Tanaka K, Itoh K: Reorganization of stress fiber-like structures in spreading platelets during surface activation.J Struct Biol. 1998; 124 (1): 13-41 PubMed Abstract | Publisher Full Text 3. Hanke J, Probst D, Zemel A, Schwarz US, et al.: Dynamics of force generation by spreading platelets.Soft Matter. 2018; 14 (31): 6571-6581 PubMed Abstract | Publisher Full Text

Is the work clearly and accurately presented and does it cite the current literature? Yes

Is the study design appropriate and is the work technically sound? Yes

Are sufficient details of methods and analysis provided to allow replication by others? Yes

If applicable, is the statistical analysis and its interpretation appropriate? Yes

Are all the source data underlying the results available to ensure full reproducibility? Yes

Are the conclusions drawn adequately supported by the results? Yes

Competing Interests: No competing interests were disclosed.

Reviewer Expertise: theoretical biophysics, cellular biophysics, mechanobiology, cell adhesion, cytoskeleton

I confirm that I have read this submission and believe that I have an appropriate level of expertise to confirm that it is of an acceptable scientific standard. 
Benny Geiger, Weizmann Institute of Science, Rehovot, Israel

\section{For Ulrich Schwarz}

Thank you Uli for the insightful comments, questions raised and suggestions.

1) Good point, we decided upon this particular set-up because we wanted to observe the platelet behavior before the addition of an agonist $\left(\mathrm{Mn}^{++}\right.$or thrombin).

2) Thanks for relating to the marginal band of microtubules. The marginal band may be seen in figure 4 as suggested, and the relevant live-cell imaging is added here. We have found a good example, addressing the question. Please see movie accessible here. We do believe we can see the MB coiling here, with an extending microtubule.

3) We didn't include it in the study. Treatment with Blebbistatin affected the organization of actin and focal adhesion like structures, but the Blebbistatin-treated platelets effect on MT extension was not tested.

4) The data is available as fig. S3, we felt it would be to cluttered to have both in one main figure.

Competing Interests: No competing interests were disclosed.

Reviewer Report 08 June 2020

https://doi.org/10.5256/f1000research.26040.r64005

(C) 2020 Yamada K. This is an open access peer review report distributed under the terms of the Creative Commons Attribution License, which permits unrestricted use, distribution, and reproduction in any medium, provided the original work is properly cited. The author(s) is/are employees of the US Government and therefore domestic copyright protection in USA does not apply to this work. The work may be protected under the copyright laws of other jurisdictions when used in those jurisdictions.

\section{Kenneth Yamada}

National Institutes of Health, National Institute of Dental and Craniofacial Research, Bethesda, MD, USA

This manuscript provides nicely quantitative descriptions of the early steps of platelet attachment to either collagen IV or fibrinogen. As such, it will provide valuable information for the field and stimulate additional research in this area, e.g., concerning the regulation of filopodial activity during spreading. There are several aspects of this excellent study that would be strengthened by the addition of either limited amounts of new data or at least some additional discussion.

1. This study focuses on platelet attachment to extracellular proteins absorbed to a substrate, presumably modeling the interaction of platelets with basement membrane or a blood clot. Although the interaction of platelets with fibrinogen has been studied extensively in 
solution after activation by molecules such as thrombin during the process of platelet aggregation, this study seems to focus on interactions with an immobilized substrate. In that regard, the authors might consider that fibrin rather than fibrinogen would be the more physiological substrate. The results of initial attachment and spreading might be different on fibrin, because it is known that platelets can interact via integrin receptors more effectively to fibrin compared to fibrinogen [e.g., see PMID 26867579 and also $29021578^{1,2}$ ].

Although this reviewer does not feel it is essential, it would be quite interesting to see whether the key findings concerning platelet attachment to fibrinogen are different when the substrate is fibrin. Alternatively, the authors could discuss this point about the types of substrate in vivo.

2. The differences in response to collagen IV and fibrinogen depending on whether they were in PRP or in a salt solution were extremely dramatic. One question that should ideally be discussed involves the physiological interpretation of the blocking of adhesion by von Willebrand factor. Does this mean that attachment to exposed collagen IV in damaged basement membranes in vivo is unlikely? Some additional discussion of these surprising findings would be helpful.

3. The images of apparent surface receptors in Figure 5 are quite impressive. However, it is not clear whether the authors can call them "surface receptors" without some additional information. For example, were morphological differences observed consistent with the folded and open erect configurations of integrins before and after platelet activation?

\section{Minor comments:}

A. In Figure 2D, the figure legend refers to "detachment" over time, yet the heat map coding seems to refer to more or fewer attachment events, so how was detachment represented in this figure?

B. In Figure 4, what were the substrates used for panel sequences A and B?

C. In Figure 7, and the text, how was a "filopodia spread platelet" defined?

D. Figure 9A, do the authors happen to have any information on which receptor was used by activated platelets to spread on collagen IV after the treatment with sn528?

E. In the summary shown in Figure 10, although this is not at all essential, the authors might consider including information on the conditions used for their platelets, specifically PLT VS. PRP-PL; if not practical, this information should be indicated in the figure legend so that readers will not assume that the platelets were compared under identical conditions on the two different substrates.

F. The dishes used in this study were presumably from MatTek rather than from MaTek.

G. Very minor technical questions: Was the concentration of thrombin used actually 0.12 $\mathrm{mg} / \mathrm{ml}$ ? What was the purpose of the glycerol added to chemically fixed platelets? 


\section{References}

1. Litvinov RI, Farrell DH, Weisel JW, Bennett JS: The Platelet Integrin aIIb $\beta 3$ Differentially Interacts with Fibrin Versus Fibrinogen.J Biol Chem. 2016; 291 (15): 7858-67 PubMed Abstract | Publisher Full Text

2. Höök P, Litvinov R, Kim O, Xu S, et al.: Strong Binding of Platelet Integrin aIIb $\beta 3$ to Fibrin Clots: Potential Target to Destabilize Thrombi. Scientific Reports. 2017; 7 (1). Publisher Full Text

Is the work clearly and accurately presented and does it cite the current literature? Yes

Is the study design appropriate and is the work technically sound?

Yes

Are sufficient details of methods and analysis provided to allow replication by others? Yes

If applicable, is the statistical analysis and its interpretation appropriate?

Yes

Are all the source data underlying the results available to ensure full reproducibility? Yes

Are the conclusions drawn adequately supported by the results? Yes

Competing Interests: No competing interests were disclosed.

Reviewer Expertise: Cell-extracellular matrix interactions, cell migration, branching morphogenesis

I confirm that I have read this submission and believe that I have an appropriate level of expertise to confirm that it is of an acceptable scientific standard.

Author Response 25 Jun 2020

Benny Geiger, Weizmann Institute of Science, Rehovot, Israel

\section{For Kenneth Yamada}

Thank you, Ken, for the insightful questions, suggestions and additions to discussion.

1) We did compare the platelet "2D adhesion" to substrate-attached fibrinogen- structures where to "3D adhesion" to fibrin fibers. In the former we can see both filopodial and lamellipodial spreading as described in great detail throughout the paper. Platelets spreading on fibrin fibers was predominantly aligned along the underlying fibers, with essentially no sign for lamellipodia. These differences can be seen here. 
2) We have addressed the point you made in the relevant section of the revised manuscript. We do not propose that attachment to exposed collagen IV in damaged basement membranes in vivo is unlikely. The difference between the in vivo setting, where flow exists, which reinforces matrix adhesion. In our work the adhesion occurs under static condition where no or very little attachment occurs. This is attributable to the fact that VWF-platelet binding is facilitated by the A1 domain of VWF, which binds to the platelet receptor GPIb/IX/V. This binding requires immobilization followed by shear force, in order to be exposed and available for binding. Since we work under static experimental conditions, this binding doesn't take place.

3) In previous studies, Ohad Medalia's group conducted gold labeling experiments to localize integrins (Dahan et al. 2018, Tamir et al. 2016) and glycoprotein $1 \mathrm{~b}$ receptors (Stivala S, et al. Haematologica 2020) within platelets. They showed that many of the densities observed by cryo-ET, in platelets protrusions, are indeed integrins. These references were introduced and added to the paper. Moreover, cryo-ET of mouse platelets lacking aIIbb3 (genetically removed) showed no densities around the plasma membrane (Sorrentino et al. in preparations).

All the minor comments were addressed. Specifically:

a) The glycerol was added to the fixation solution in order to provide a protective environment around the sensitive platelet filopodia.

b) The 'filopodia spread platelet' is a platelet with filopodia that does not display lamellipodial extensions and stays in this shape for the entire experiment duration.

Competing Interests: No competing interests were disclosed.

Reviewer Report 01 June 2020

https://doi.org/10.5256/f1000research.26040.r64004

(C) 2020 Citi S. This is an open access peer review report distributed under the terms of the Creative Commons Attribution License, which permits unrestricted use, distribution, and reproduction in any medium, provided the original work is properly cited.

\section{Sandra Citi}

Department of Cell Biology, Faculty of Sciences, University of Geneva, Geneva, Switzerland

This is an interesting study on the initial phases of adhesion and spreading of platelets on two different substrates, collagen IV and fibrinogen. The authors report that on collagen IV a short-term phase of filopodial extension is followed by lamellipodia based-spreading, correlating with the insertion of 1-2 microtubules at the core of the filopodium. On fibrinogen, in contrast, there was only partial filopodia-to-lamellipodia transition, and microtubule extension was not 
observed. The quantification and image analysis of platelet spreading based on interference reflection microscopy, allowing a temporal profiling of attachment vs detachment and cryo-EM data are particularly interesting. The methods are state-of-the art, the results convincing, and the conclusions are sound.

The results should be discussed in the context of previous work on the distribution of microtubules in resting platelets and platelet filopodia (Patel-Hett et al. Blood, 2008 ${ }^{1}$ ).

Some of the Figures should be improved for clarity. Figure 1: label panels A-C on the sides. Figure 3 D-E: label panels on the side (D- collagen, E-fibrinogen). Figures 7, 8 and 9: please use dot plots and not bar-graphs. Figure 10 label Panels (A and B), and schematic panel (left) for fibrinogen, to make the difference clearer (only collagen is shown).

In the overview of platelet temporal functional interplay (Fig 10) it is not clear why the lamellipodia shape phase is there, since the filopodia-to-lamellipodia transition is partial?

Typos: $4^{\circ} \mathrm{C}$ not $4 \mathrm{C}^{\circ}$ in first paragraph of Methods, by ligand not byligand in 5 th paragraph Discussion.

\section{References}

1. Patel-Hett S, Richardson JL, Schulze H, Drabek K, et al.: Visualization of microtubule growth in living platelets reveals a dynamic marginal band with multiple microtubules.Blood. 2008; 111 (9): 4605-16 PubMed Abstract | Publisher Full Text

Is the work clearly and accurately presented and does it cite the current literature? Partly

Is the study design appropriate and is the work technically sound?

Yes

Are sufficient details of methods and analysis provided to allow replication by others? Yes

If applicable, is the statistical analysis and its interpretation appropriate?

I cannot comment. A qualified statistician is required.

Are all the source data underlying the results available to ensure full reproducibility? Yes

Are the conclusions drawn adequately supported by the results?

Yes

Competing Interests: No competing interests were disclosed.

Reviewer Expertise: Cell-cell junctions, cytoskeleton.

I confirm that I have read this submission and believe that I have an appropriate level of 
expertise to confirm that it is of an acceptable scientific standard.

\section{Author Response 25 Jun 2020}

Benny Geiger, Weizmann Institute of Science, Rehovot, Israel

\section{For Sandra Citi}

Thank, Sandra, for the useful comments, suggestions, corrections and thoughts.

1) We have further addressed, in the discussion MTs organization and dynamics in platelets, with the relevant reference.

2) We have added labels in fig. 10 ( $A$ and $B$ ) and we have referred to the difference between collagen and fibrinogen in the figure legend more clearly. Since the figures would be very similar we thought it to be better to address the issue in this manner.

3) Typos were fixed

Competing Interests: No competing interests were disclosed.

The benefits of publishing with F1000Research:

- Your article is published within days, with no editorial bias

- You can publish traditional articles, null/negative results, case reports, data notes and more

- The peer review process is transparent and collaborative

- Your article is indexed in PubMed after passing peer review

- Dedicated customer support at every stage

For pre-submission enquiries, contact research@f1000.com 